\title{
Simultaneous Orbit and Atmospheric Density Estimation for a Satellite Constellation
}

\author{
Joanna C. Hinks ${ }^{*}$ and Mark L. Psiaki ${ }^{\dagger}$ \\ Cornell University, Ithaca, NY 14853
}

\begin{abstract}
A method is defined for simultaneous atmospheric density calibration and satellite orbit determination for a satellite constellation, and a linearized observability analysis is performed to evaluate the feasibility of the approach. Such an estimation scheme will provide data-based estimates of upper atmosphere density that improve on existing models, e.g. the Jacchia models, along with enhanced accuracy of the associated satellites' orbits. A new spline-based atmospheric density parameterization is developed that meshes well with the structure of the orbit determination problem and can be initialized so as to match the outputs of a traditional model. While conceptually similar to previous atmospheric calibration efforts, the proposed constellation approach restricts its global density estimates to the relevant satellite altitude range and thus reduces the complexity of the estimation problem. Measurements include dual-one-way-ranging between pairs of adjacent satellites in the same orbital plane, and carrier phase and pseudorange measurements between ground stations and satellites. Equations for a linearized observability analysis are derived and shown to be equivalent to the covariance calculations of an extended square-root information filter. In addition to the system observability evaluation, the impact of incorporating some a priori density information is explored. The results show that atmospheric density can be observed, but a reasonable amount of a priori information is necessary to obtain useful estimates. Degree of observability depends on the constellation configuration and dynamic model parameter values.
\end{abstract}

\section{Introduction}

$\mathrm{O}$ RBIT determination is the process by which satellite trajectories are estimated. The available measurements are combined to yield a history of the satellite's position and velocity. Accuracy of orbit estimates is limited by the quality, quantity, and types of available measurements, and by the accuracy of the dynamic model assumed for the satellite's motion. Thus if an application requires high precision, one normally needs high quality measurements and models.

For many satellites in low earth orbit (LEO), the largest dynamic model uncertainty stems from atmospheric $\operatorname{drag}^{1}$. Acceleration due to atmospheric $\operatorname{drag} \boldsymbol{a}_{D}$ is related to atmospheric density $\rho$ by the equation

$$
\boldsymbol{a}_{D}=-\frac{1}{2}\left(C_{D} \frac{A_{v}(t)}{m_{s}}\right) \rho v_{r}^{2} \boldsymbol{e}_{v}
$$

where $C_{D}$ is a drag coefficient, $A_{v}(t)$ is the cross-sectional area of the satellite in the direction of travel, $m_{s}$ is the total spacecraft mass, $v_{r}$ is the velocity magnitude relative to the ambient atmosphere, and $\boldsymbol{e}_{v}$ is a unit vector in the relative velocity direction. Uncertainty enters this equation in three ways. First, the scalar product $\left(C_{D} \frac{A}{m_{s}}\right)$, known as the inverse ballistic coefficient, is generally uncertain and may be time-varying. Second, the relative velocity may be uncertain, either because it has not yet been estimated accurately or because the local wind does not rotate perfectly with the Earth. Finally, atmospheric density is very difficult to determine. Three basic paradigms exist for dealing with drag uncertainty: It can be modeled, measured directly or indirectly, or estimated in conjunction with satellite orbits.

\footnotetext{
* Ph.D. Candidate, Sibley School of Mechanical and Aerospace Engineering, 127 Upson Hall, Student Member, AIAA.

${ }^{\dagger}$ Professor, Sibley School of Mechanical and Aerospace Engineering, 206 Upson Hall. Associate Fellow, AIAA. 
Models of atmospheric density have been developed and studied widely in the past 50 years. The Jacchia series of models assumes a temperature profile, and integrates the barometric and diffusion differential equations upwards to the desired altitude. A number of empirical corrections are also applied based on satellite orbit data ${ }^{2,3,4,5}$. The Mass Spectrometer and Incoherent Scatter (MSIS) series of models has been derived based on data from science instruments. This series simultaneously computes temperature and density by evaluating a complicated set of functions involving hundreds or thousands of coefficients ${ }^{6,7,8}$. While these two series of density models are the most common, many others have been developed by various researchers. All of them, however, retain fairly large levels of uncertainty even after decades of development. Typical model standard deviations start at 10-20\% under normal conditions and can be much greater at times of unusually high or low solar and geomagnetic activity ${ }^{9,10,11}$. Much of this inaccuracy comes from the inadequacy of currently available metrics of solar activity, which do not fully capture the effects of the sun on the atmosphere ${ }^{12,13}$. Most models take as inputs the $10.7 \mathrm{~cm}$ solar flux $F_{10.7}$ and the 3-hourly geomagnetic index $K_{p}$ (or its equivalent $a_{p}$ ), which are reasonable but incomplete metrics. Furthermore, there is still a great deal to be learned about the complex dynamics of the atmosphere, and good measurements are a limiting factor. It is unlikely that modeling efforts will improve significantly in the near future.

The second approach to the atmospheric density problem depends on highly accurate measurements or targeted sensors to remove the effects of atmospheric drag from the orbit solution. For instance, satellite missions like CHAMP and GRACE carry high-quality accelerometers to estimate non-gravitational perturbations ${ }^{14}$. Special sensors on Dynamics Explorer 2 made in situ measurements of temperature and atmospheric composition, which were later used to develop the MSIS models ${ }^{15}$. Increasingly, new satellite missions carry GPS receivers, which support tracking of satellite motion to within centimeters and thus reduce or eliminate the need for accurate models of dynamic perturbations ${ }^{16}$. There are even a few missions, such as Gravity Probe B, which eliminate drag perturbations entirely by flying a proof mass in a vacuum chamber and actively steering the spacecraft to follow its drag-free motion ${ }^{17}$. While all of these techniques are effective, they can be implemented only with expensive, dedicated, space-born instrumentation.

When atmospheric models are not sufficiently accurate and special flight instruments are not an option, one can attempt to estimate satellite drag or density parameters along with the orbits. In particular, atmospheric calibration efforts attempt to estimate global density parameters or density corrections on the basis of some form of satellite data. The density estimates can then be applied to the orbits of other spacecraft as well. Several calibration projects have recently demonstrated improved density knowledge and orbit determination performance in comparison with standard models. For example, some approaches take as their measurements the two-line elements (TLE's) of tracked space objects, which are made available by NORAD, and employ long histories of these measurements to calculate corrections to a density model ${ }^{18,19,20,21}$. Another method's calibration strategy aims to reduce the standard deviation of many months' worth of multi-day batch fits of a satellite's inverse ballistic coefficient ${ }^{12,13}$. The Air Force Space Battlelab has developed the High Accuracy Satellite Density Model (HASDM), which incorporates Space Surveillance Network (SSN) data from a set of frequently tracked calibration satellites to both estimate and predict global atmospheric density ${ }^{22,23}$. All of these methods have shown promise in reducing orbit determination fit errors for satellites not included in the calibration set.

This paper proposes a new form of atmospheric calibration to support precision orbit determination for a satellite constellation. Its approach attempts to simultaneously estimate the satellite orbits along with the density profile for the altitude region spanned by the constellation. It employs the preexisting radiocommunication signals of the constellation as its measurements, rather than dedicated tracking data. Significantly, none of the signals were designed for navigation, yet the information in the data set exceeds what has typically been available for density estimation.

The candidate constellation would have 50-100 satellites arranged in several evenly spaced orbital planes, with nearly circular, low-earth orbits. Each satellite in an orbital plane would encounter similar density variations to those experienced by the others in that plane, thus providing a multiplicity of measurements for a given density feature. Adjacent satellite pairs would be joined by crosslink range signals, and additional signals would measure ranges between the satellites and a set of ground stations. For the envisioned system, sub-meter orbit accuracy is desired throughout the orbit, so the density must be parameterized in a way that provides sufficient spatial and temporal resolution ${ }^{24}$. A spline-based density parameterization method has been developed for this purpose.

The goal of this paper is to study the proposed atmospheric calibration/orbit-determination scheme, particularly with respect to observability. Specifically, it seeks to answer the question, "Is the available data sufficient to simultaneously estimate the orbits of all satellites in the constellation, as well as the atmospheric density spline parameters?" One would not expect the system to be fully observable, due to the large number of parameters to be estimated and due to coupling of certain parameters' effects. However, this estimation scheme can be used in a recursive (Kalman) filtering context, where a priori estimates are available from physics or past efforts. In such a 
scenario, an observability analysis can still yield information about which parts of the a priori estimate can be improved with new data and which quantities should be more carefully modeled because the measurements add no further knowledge.

This paper offers three main contributions. First, it develops a spline-based density parameterization method which is well-suited to the constellation estimation context. Second, it defines and elaborates the problem of performing atmospheric calibration using a constellation of satellites. Finally, a linearized observability analysis is performed to determine the feasibility of the proposed approach and to identify potential areas of weakness.

The rest of this paper is organized as follows: Section II gives an overview of the spline-based density parameterization technique and some of the issues related to representation of density. In Section III, the proposed estimation problem is formulated, and important assumptions about the dynamics and measurements of the system are clarified. Section IV considers the issue of observability, and derives the equations for the linearized observability analysis. Results of the observability analysis are given in Section V along with some suggested alternative tests and their results. Section VI presents the paper's conclusions and suggests areas for future study.

\section{Spline-Based Density Parameterization}

In order to estimate an atmospheric density distribution, one must first decide how to represent it. This choice will guide the development of the estimation scheme. Some past efforts have used altitude-dependent corrections to the outputs of a Jacchia model ${ }^{19}$. Another parameterization makes corrections to the model inputs, for instance by specifying the coefficients of a spherical harmonic expansion for exospheric temperature ${ }^{22}$ above. The chosen method must be capable of representing a complex density distribution and be appropriate for the specific purposes in mind.

The chosen parameterization takes the form

$$
\rho\left(\phi, \lambda, h ; \boldsymbol{p}_{a}\right)=\rho_{0}\left(\phi, \lambda ; \boldsymbol{p}_{a}\right) \exp \left(-\frac{h-h_{0}(\phi)}{H_{\text {scale }}\left(\phi, \lambda ; \boldsymbol{p}_{a}\right)}\right)
$$

In this equation, the inputs are latitude $\phi$, longitude in a sun-fixed frame $\lambda$ (essential the angular form of local solar time), altitude $h$, and a parameter vector $\boldsymbol{p}_{a}$, with $\phi$ and $h$ defined relative to an ellipsoid. For each latitude and longitude, density has a value $\rho_{0}$ at a nominal altitude $h_{0}$, and above and below this nominal altitude it varies exponentially according to the scale height $H_{\text {scale. }}$. Both $\rho_{0}$ and $H_{\text {scale }}$ are splined functions of latitude and longitude. The parameter vector $\boldsymbol{p}_{a}$ stores the values (and spatial derivatives) of $\rho_{0}$ and $H_{\text {scale }}$ at particular grid points, and cubic spline interpolation is used to ensure smooth variation between these points. Given a suitable number of grid points, this parameterization will be comparable to a spherical harmonic representation. It has been chosen because of the ease with which various of its partial derivatives can be calculated. A detailed description and equations of the spline interpolation, as well as other aspects of this parameterization, are given in the appendix at the end of this paper.

It is widely recognized that Earth's atmosphere is only approximately exponential in density. This exponential assumption is valid, however, over a small range of altitudes. For the nearly-circular orbits of a candidate satellite constellation, the altitudes encountered fall well within the acceptable range. This has been verified by determining altitude variations resulting from both actual satellite orbit eccentricities and the effects of Earth's ellipsoidal shape. Within the determined range, evaluations of standard atmospheric models such as NRLMSISE-00 ${ }^{8}$ show density variations that are practically indistinguishable from the exponential form. Because this parameterization is specifically intended for a satellite constellation application, it does not need to represent densities of the full atmosphere; a thin shell at the right altitude is adequate. The nominal altitude $h_{0}(\phi)$ falls approximately in the center of this shell, and its latitude dependence adjusts for the curvature of the WGS-84 ellipsoid relative to the nominal constellation altitude.

A natural way to visualize the spline-based density parameterization is as a two-dimensional Cartesian grid of points in the variables $(\phi, \lambda)$, with a surface stretched between them. As countless historical mapmakers can attest, a number of mathematical difficulties arise from the representation of a sphere as a rectangular grid. For instance, the mapping is not one-to-one; when one reaches the far "eastern" edge of the grid, where $\lambda=180^{\circ}$, this is really the same location as the corresponding point on the "western" edge, where $\lambda=-180^{\circ}$. At the poles, the situation is further complicated because, regardless of the longitude, every point with $\phi=90^{\circ}$ represents the "North pole", and every point with $\phi=-90^{\circ}$ represents the "South pole". These singularities give rise to a number of constraints on the valid node point values of the spline parameterization. Other constraints are entailed by the physical

American Institute of Aeronautics and Astronautics 
requirement that the density vary "smoothly" over the globe, including at the poles. This second group of constraints influences the spatial derivative values that are also stored as part of the parameterization. The appendix contains further details of the constraints and their implementation.

All the density information at the constellation altitude can be captured by the values in the parameter vector $\boldsymbol{p}_{a}$, along with the spline algorithms that extract $\rho_{0}(\phi, \lambda)$ and $H_{\text {scale }}(\phi, \lambda)$ from $\boldsymbol{p}_{a}$. As described above, the information stored in this vector is a list of density and scale height values at certain $(\phi, \lambda)$ points, and also the first partial derivatives of those values with respect to latitude and longitude and the cross-partial-derivative with respect to both latitude and longitude. Thus, there are eight scalar pieces of information for each distinct $(\phi, \lambda)$ point: Four associated with density, $\left\{\rho_{0}, \frac{\partial \rho_{0}}{\partial \phi}, \frac{\partial \rho_{0}}{\partial \lambda}, \frac{\partial^{2} \rho_{0}}{\partial \lambda \partial \phi}\right\}$, and four associated with scale height, $\left\{H_{\text {scale }}, \frac{\partial H_{\text {scale }}}{\partial \phi}, \frac{\partial H_{\text {scale }}}{\partial \lambda}\right.$, $\left.\frac{\partial^{2} H_{\text {scale }}}{\partial \lambda \partial \phi}\right\}$. These values are all stacked together to form the vector $\boldsymbol{p}_{a}$. Although the order of the elements might change, a segment of this vector would look like

$$
\boldsymbol{p}_{a}=\left[\begin{array}{c}
\vdots \\
\rho_{0}\left(\phi_{i}, \lambda_{j}\right) \\
\frac{\partial \rho_{0}}{\partial \phi}\left(\phi_{i}, \lambda_{j}\right) \\
\frac{\partial \rho_{0}}{\partial \lambda}\left(\phi_{i}, \lambda_{j}\right) \\
\frac{\partial^{2} \rho_{0}}{\partial \lambda \partial \phi}\left(\phi_{i}, \lambda_{j}\right) \\
H_{\text {scale }}\left(\phi_{i}, \lambda_{j}\right) \\
\frac{\partial H_{\text {scale }}}{\partial \phi}\left(\phi_{i}, \lambda_{j}\right) \\
\frac{\partial H_{\text {scale }}}{\partial \lambda}\left(\phi_{i}, \lambda_{j}\right) \\
\frac{\partial^{2} H_{\text {scale }}}{\partial \lambda \partial \phi}\left(\phi_{i}, \lambda_{j}\right) \\
\vdots
\end{array}\right]
$$

for a general grid point $\left(\phi_{i}, \lambda_{j}\right)$ that is not at one of the poles.

The length of the parameter vector is determined by the number of latitude and longitude grid points, that is, the desired resolution of the estimated density field. Typically, there are 150 to 300 scalar parameters to be estimated. While this number may seem high, it is manageable. A satellite constellation may have close to 1000 values to be estimated just for the satellite orbital states and a few satellite-specific parameters. Therefore, an extra 200 atmospheric density parameters may be reasonable.

A second concern for a large number of atmospheric parameters, and the main topic of this paper, is that of their observability. Even with incomplete observability an operational filter could still function well. A recursive estimator would start with some a priori information on density and improve on that initial guess. It would leave unobservable parameter combinations at their a priori values.

While the preceding description explains how a global density field can be stored, it provides no connection, theoretical or empirical, to the physics of the atmosphere. Even if the estimation technique does not require an initial guess, one desires some reassurance that results are realistic. Furthermore, nonlinear problems require a sufficiently close initial estimate in order for linearization to be valid. These needs are met by an initialization procedure that accepts any set of density "truth" data and outputs a corresponding vector $\boldsymbol{p}_{a}$. "Truth" data can come from standard atmospheric models, or from satellite-carried sensors or any other source, as long as the density data points are globally distributed. In order to estimate scale heights, some altitude diversity within the range of interest is necessary. After one chooses the desired latitude and longitude resolution, the initialization procedure performs a least-squares fit to find the $\boldsymbol{p}_{a}$ values that most nearly cause the interpolated spline to match the data points. This strategy has been found to fit "standard" density models closely with surprisingly few latitude and longitude divisions. 
For instance, Figure 1 shows a fit of a splined parameterization with five latitude points and six longitude points. The fit is to the NRLMSISE00 model, during average solar and geomagnetic conditions. The surface is the cubic-splineinterpolated manifold corresponding to the solved-for $\boldsymbol{p}_{a}$ parameters, and the points are the model's "data". For this particular fit, the mean density relative error between the data and the splined parameterization was $0.51 \%$, and the maximum relative error was $4.22 \%$. This fit involves only 156 parameters and fits 3000 data points. Note: Figure 1 depicts the splined density only at the nominal altitude. Its displayed data points are artificially mapped to that altitude by means of the splined scale height.

In order to use a particular density parameterization for estimation, one must be able to

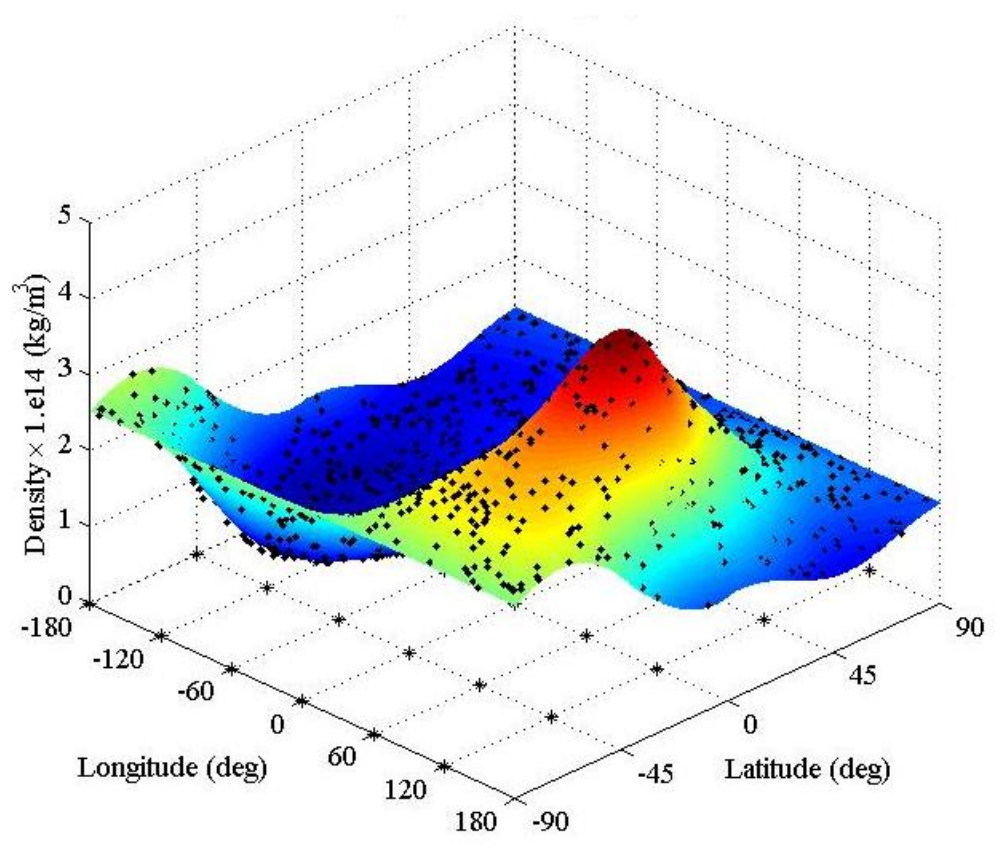

Figure 1. Density initialization data from NRLMSISE-00 and the corresponding best-fit splined surface derive its partial derivatives with respect to the elements of the filter's estimated state. The partial derivatives provide information about the way small changes in one quantity affect some other quantity of interest. For this scenario, estimation requires the partial derivatives of density with respect to the inertial position vector $\boldsymbol{r}_{I C R S}(x, y, z$ position, not $\phi, \lambda, h$ position) and with respect to the parameter vector $\boldsymbol{p}_{a}$. Derivatives of the spline-parameterized quantities $\rho_{0}$ and $H_{\text {scale }}$ with respect to $\phi, \lambda$, and the elements of $\boldsymbol{p}_{a}$ can be computed using minor modifications of the interpolation algorithm. Thus the quantities $\frac{\partial \rho_{0}}{\partial \phi}, \frac{\partial H_{\text {scale }}}{\partial \phi}, \frac{\partial \rho_{0}}{\partial \lambda}, \frac{\partial H_{\text {scale }}}{\partial \lambda}, \frac{\partial \rho_{0}}{\partial \boldsymbol{p}_{a}}$, and $\frac{\partial H_{\text {scale }}}{\partial \boldsymbol{p}_{a}}$ are readily available. The desired partial derivatives $\frac{\partial \rho}{\partial \boldsymbol{r}_{I C R S}}$ and $\frac{\partial \rho}{\partial \boldsymbol{p}_{a}}$ are then computed by some simple calculations and repeated application of the chain rule. The appendix elaborates further on this topic for the interested reader.

\section{Formulation of the Estimation Problem}

The problem of determining satellite orbits and atmospheric density parameters from noisy data falls within the scope of estimation theory. Estimation is the process by which one extracts desired information from measurements in some optimal fashion. To employ standard estimation algorithms, two sets of mathematical models must be created. First, there are differential or difference equations that model the dynamic process of interest. These equations describe the evolution of the system state vector, where the states are the quantities to be estimated, such as position, velocity, or time-varying parameters. Second, estimation methods need mathematical models of the measurements, and specifically of the functional relationship between the state vector and the measurements. A final requirement is a statistical description of all uncertainties in the system. The dynamics models include a stochastic contribution from process noise, and the measurements models typically have additive noise representative of sensor errors. Any correlations between process and measurement noise must be accurately described.

\section{A. State vector and dynamics models}

The state vector for this estimation scheme contains many components. First, there are ten states associated with each satellite. For the $j^{t h}$ satellite, they are contained in the vector $\boldsymbol{x}_{s}^{j}$, which will be discussed in more detail below. In addition to states relating to individual satellites, the state vector includes the atmospheric density parameter vector $\boldsymbol{p}_{a}$, which will also be estimated. The last part of the state vector contains a set of "nuisance states" that must 
be estimated but are not actually of interest. These are unknown real-valued biases on accumulated delta range phase measurements, and include two distinct sets of biases: $\boldsymbol{b}_{c l}$ and $\boldsymbol{b}_{g s}$. The vector $\boldsymbol{b}_{c l}$ contains biases on crosslink measurements between satellites, and $\boldsymbol{b}_{g s}$ contains biases on measurements between ground stations and satellites. These measurement quantities are explained in more depth later in Section III. When all these states are stacked, the dynamic state vector becomes

$$
\boldsymbol{x}=\left[\begin{array}{c}
\boldsymbol{x}_{s}^{1} \\
\boldsymbol{x}_{s}^{2} \\
\vdots \\
\boldsymbol{x}_{s}^{M} \\
\boldsymbol{p}_{a} \\
\boldsymbol{b}_{c l} \\
\boldsymbol{b}_{g s}
\end{array}\right]
$$

where $\boldsymbol{x}$ is the full system state and $M$ is the number of satellites in the constellation. In its general form, the nonlinear dynamics differential equation for this state vector can be written as

$$
\dot{\boldsymbol{x}}(t)=f(t ; \boldsymbol{x}(t), \boldsymbol{w}(t))
$$

where $f$ defines the time evolution of all the states, and $\boldsymbol{w}(t)$ is the system process noise vector. The discretized version of this function can be written as

$$
\boldsymbol{x}_{k+1}=\boldsymbol{f}\left(k ; \boldsymbol{x}_{k}, \boldsymbol{w}_{k}\right)
$$

where $\boldsymbol{x}_{k}$ is the state at the $k^{\text {th }}$ sample time $t_{k}$, and $\boldsymbol{w}_{k}$ parameterizes $\boldsymbol{w}(t)$ during the interval from $t_{k}$ to $t_{k+1}$. The function $\boldsymbol{f}\left(k ; \boldsymbol{x}_{k}, \boldsymbol{w}_{k}\right)$ is computed in practice via numerical integration of differential Eq. (5), starting from the initial condition $\boldsymbol{x}_{k}=\boldsymbol{x}\left(t_{k}\right)$. The general function $\mathfrak{f}$ given in Eq. (5) can be broken down into specific functions for the distinct components of the state vector.

Each individual satellite $j$ has a set of states of the form

$$
\boldsymbol{x}_{S}^{j}=\left[\begin{array}{c}
\boldsymbol{r}_{s}^{j} \\
\boldsymbol{v}_{s}^{j} \\
\delta t_{R}^{j} \\
\delta f_{R}^{j} \\
\beta_{D}^{j} \\
\beta_{S}^{j}
\end{array}\right]
$$

where $\boldsymbol{r}_{s}^{j}$ is the satellite Cartesian position vector in an inertial frame, $\boldsymbol{v}_{s}^{j}$ is the corresponding velocity vector, $\delta t_{R}^{j}$ and $\delta f_{R}^{j}$ are the satellite clock error and rate error, respectively, and $\beta_{D}^{j}$ and $\beta_{S}^{j}$ are the inverse ballistic coefficient and the solar radiation pressure coefficient for that particular satellite.

Dynamic behavior for each satellite's position and velocity is governed by Newton's second law: acceleration is proportional to force divided by mass. This leads to the equation

$$
\left[\begin{array}{c}
\dot{\boldsymbol{r}}_{s}^{j}(t) \\
\dot{\boldsymbol{v}}_{S}^{j}(t)
\end{array}\right]=\left[\begin{array}{c}
\boldsymbol{v}_{s}^{j}(t) \\
\boldsymbol{f}_{S}\left(t ; \boldsymbol{r}_{s}^{j}, \boldsymbol{v}_{s}^{j}, \boldsymbol{p}_{a}, \beta_{D}^{j}, \beta_{S}^{j}\right)
\end{array}\right]
$$

Note that no process noise enters the satellite dynamics directly. This analysis assumes that the dominant errors in the dynamic model enter it through $\boldsymbol{p}_{a}$. The total force $\mathfrak{f}_{s}$ consists of summed contributions from Earth's gravity, the gravitational attractions of the Sun and Moon, atmospheric drag, and solar radiation pressure. A simplified Earth gravity model with only a small number of terms will be used for this analysis. While additional forces and more complicated force models would certainly be included in an actual orbit determination run, neglecting them here will not significantly degrade the results of the observability analysis ${ }^{25}$. 
In addition to its position and velocity dynamics, each satellite has dynamic models associated with its clock. A standard clock error model is used ${ }^{26}$. It takes the form

$$
\left[\begin{array}{c}
\dot{\delta t_{R}^{j}}(t) \\
\dot{\delta f_{R}^{j}(t)}
\end{array}\right]=\left[\begin{array}{ll}
0 & 1 \\
0 & 0
\end{array}\right]\left[\begin{array}{l}
\delta t_{R}^{j}(t) \\
\delta f_{R}^{j}(t)
\end{array}\right]+\left[\begin{array}{c}
w_{\phi}^{j}(t) \\
w_{f}^{j}(t)
\end{array}\right]
$$

where $w_{\phi}^{j}$ and $w_{f}^{j}$ are phase and frequency process noise that drive the clock error. The statistical properties of the process noise are chosen based on the particular type of clock carried by the satellite. These clock errors affect the phases of the transmitted crosslink and downlink signals, and the crosslink receiver's measured phase outputs.

Accelerations due to atmospheric drag and solar radiation pressure depend not only on environmental variables but also on the shape, surface properties, and mass distribution of specific satellites. Generally, these effects are captured by proportionality constants that multiply the relevant vector quantities. Both inverse ballistic coefficient $\beta_{D}^{j}$ and solar radiation pressure coefficient $\beta_{S}^{j}$ have dimensions of area divided by mass:

$$
\left[\begin{array}{c}
\beta_{D}^{j} \\
\beta_{S}^{j}
\end{array}\right]=\left[\begin{array}{l}
C_{D} \frac{A_{v 0}}{m_{s}} \\
C_{R} \frac{A_{S 0}}{m_{s}}
\end{array}\right]
$$

where $A_{v 0}$ and $A_{s 0}$ are the average projected areas perpendicular, respectively, to the velocity and sun directions. The total spacecraft mass is $m_{s}$, and $C_{D}$ and $C_{R}$ are nondimensional scalars. In situations where the projected areas vary rapidly, a priori models of these variations, $A_{v}(t)$ and $A_{s}(t)$, can be incorporated into the force models in $f_{s}$ via the ratios $A_{v}(t) / A_{v 0}$ and $A_{s}(t) / A_{s 0}$. Such models preserve the nearly constant nature of $\beta_{D}^{j}$ and $\beta_{S}^{j}$. Due to the remaining uncertainty, the states $\beta_{D}^{j}$ and $\beta_{S}^{j}$ are modeled as either constants or slowly-varying random walks:

$$
\left[\begin{array}{c}
\dot{\beta}_{D}^{j} \\
\dot{\beta}_{S}^{j}
\end{array}\right]=\left[\begin{array}{c}
w_{D}^{j} \\
w_{S}^{j}
\end{array}\right]
$$

where $w_{D}^{j}$ and $w_{S}^{j}$ are continuous time white noise for a random walk, or zero for constants. The joint observability of these states and the atmospheric density states is a subject of investigation ${ }^{27}$.

After ten states for every satellite in the constellation, the system state vector contains a sub-vector $\boldsymbol{p}_{a}$ of density parameters. As the spline-based atmospheric density parameterization does not incorporate any physics, it is difficult to say how its dynamics should be modeled. Empirically, the diurnal variation is the primary effect, but this component has been removed by specifying the spline's longitude grid relative to local solar time. Some diurnal effects remain, however, due to rotation of the Earth-fixed magnetic field. Other well-documented variations include effects of the 11-year solar cycle and the 27-day solar rotation period, variations due to specific solar activity, variations caused by geomagnetic activity, a semiannual variation, and what are known as seasonallatitudinal variations ${ }^{3}$. With the exception of the solar and geomagnetic activity effects, all of these components act on much longer time scales than those envisioned for the estimation problem, and thus enter the problem mostly as biases that would be included in the initialization procedure. One goal of this observability analysis is to determine if the available data are sufficient to estimate the remaining short-term variations due to solar activity, geomagnetic activity, and other random fluctuations.

In the absence of a reliable physics-based model for these effects, the dynamics of the density parameter vector $\boldsymbol{p}_{a}$ is modeled as a first-order Markov process, where each element evolves according to

$$
\dot{p}_{a i}=-\frac{1}{\tau_{a i}}\left(p_{a i}-p_{a 0 i}\right)+w_{a i}
$$

where $\tau_{a i}$ is the Markov time constant, $p_{a 0 i}$ is an a priori estimate of the $i^{\text {th }}$ element $p_{a i}$, and $w_{a i}$ is the continuous time process noise. Typically $p_{a 0 i}$ is the initial value determined from a fit to a standard model as in Figure 1 . Proper tuning of $\tau_{a i}$ and the process noise intensity will prevent $p_{a i}$ from reverting to the a priori standard model if the data dictate otherwise. 
Time constants for the process have been determined empirically from one of the standard models such as Jacchia 71 or NRLMSISE-00. This was done by assuming different input histories to the model, representative of mild, moderate, and severe solar and geomagnetic disturbances. For each set of inputs, the initialization procedure yielded the corresponding $\boldsymbol{p}_{a}$, and the way in which the values in $\boldsymbol{p}_{a}$ change with time was leveraged to select appropriate process time constants and reasonable process noise intensities. Situations are considered where time constants and process noise intensities can change over time in response to changes in geomagnetic or solar activity, as described in Ref. 28.

In a typical batch observability analysis, process noise is neglected altogether in dynamic models, although its effects may be characterized by studying the results of different batch interval lengths. If a relatively short batch interval gives good observability, a higher level of process noise is acceptable. This paper incorporates process noise directly, as will be explained in Section IV. The inclusion of process noise does not change the theoretical observability of a system; rather, it provides a more realistic relative observability of the states.

The final part of the state vector contains all the measurement biases. Although their particular values are not important, measurement biases must be estimated as part of the state vector in order to make optimal use of the available accumulated delta range measurements. They are modeled as constants by the equation

$$
\left[\begin{array}{l}
\dot{\boldsymbol{b}}_{c l} \\
\dot{\boldsymbol{b}}_{g S}
\end{array}\right]=\left[\begin{array}{l}
0 \\
0
\end{array}\right]
$$

The chief difficulty arising from the biases is their number. For every pair of adjacent satellites with dual oneway ranging, there are two crosslink signals and distinct biases for each crosslink. Every time a satellite comes within view of a ground station, a new bias from the resulting measurement gets added to the state vector. This bias is constant for the entire pass of the satellite over that ground station, but if the ground station stops tracking the satellite and then resumes tracking on another pass, a new bias applies to the measurement. Any satellite might pass over any ground station any number of times, so the "obsolete" states in $\boldsymbol{b}_{g s}$ must be removed to keep the size manageable.

\section{B. Measurement Models}

Estimation problems also require mathematical models of the way in which the system state affects the measurements. These are necessary in order to optimally extract information about the system states from measurements which are indirectly related to those states, noisy, or biased. Three types of measurements are available for the proposed constellation orbit determination scheme. First, there are dual-one-way-ranging measurements between the adjacent satellites in an orbital plane, called crosslinks. Each satellite of a pair measures a phase-based accumulated delta range relative to the other satellite, with a unique unknown bias in each direction. Next, there is an accumulated delta range between each ground station and every satellite it tracks at a given time. This delta range also includes an unknown bias. Finally, there are GPS-like pseudorange measurements between the ground stations and satellites. Although the pseudoranges are not biased, they are much noisier than the corresponding accumulated delta ranges.

The crosslink measurements are formed as a beat phase: when a signal arrives from an adjacent satellite, its phase is compared to the phase of the receiver satellite's onboard oscillator. In this way it forms a biased range measurement between the transmitting satellite's position at the true time of transmission, $t_{\text {trans }}$, and the receiving satellite's position at the true time of reception, $t_{\text {meas }}$. Thus the equation for the crosslink accumulated delta range between transmitting satellite $\ell$ and receiving satellite $j$ is given by

$$
\lambda \phi^{j \ell}=\sqrt{\left[\boldsymbol{r}_{s}^{j}\left(t_{\text {meas }}\right)-\boldsymbol{r}_{s}^{\ell}\left(t_{\text {trans }}\right)\right]^{\mathrm{T}}\left[\boldsymbol{r}_{s}^{j}\left(t_{\text {meas }}\right)-\boldsymbol{r}_{s}^{\ell}\left(t_{\text {trans }}\right)\right]}+c\left(\delta t_{R}^{j}\left(t_{\text {meas }}\right)-\delta t_{R}^{\ell}\left(t_{\text {trans }}\right)\right)+\lambda b^{j \ell}+\eta^{j \ell}
$$

where $\lambda \phi^{j \ell}$ is the accumulated delta range, converted to distance by the wavelength $\lambda, \boldsymbol{r}_{s}^{j}\left(t_{\text {meas }}\right)$ is the position vector of the receiving satellite at the true time of measurement, $\boldsymbol{r}_{s}^{\ell}\left(t_{\text {trans }}\right)$ is the position vector of the transmitting satellite at the true time of transmission, $\delta t_{R}^{j}$ and $\delta t_{R}^{\ell}$ are the receiving and transmitting clock errors, respectively, $\lambda b^{j \ell}$ is the bias converted to an equivalent distance, and $\eta^{j \ell}$ is noise. Delays due to neutral atmosphere are nonexistent at satellite altitudes, and ionospheric effects are small. 
While the physical measurement model is formed based on the positions at the true times of transmission and reception, the receiver reports only the reception time according to its own internal clock, $t_{R}^{j}$, and the transmitter reports no time. This leaves the estimation algorithm the following challenge: It needs to use $t_{\text {meas }}$ and $t_{\text {trans }}$ as part of the measurement model by which it estimates the state. These two times, however, must be inferred from state vector elements. This inference is carried out by solving implicit constraint equations. Thus, there is a seeming paradox of having to know the state in order to estimate it. One starts to resolve this paradox by considering the implicit equations for $t_{\text {meas }}$ and $t_{\text {trans }}$ :

$$
\begin{gathered}
0=g_{1}\left(t_{\text {meas }} ; \boldsymbol{x}_{k}, \boldsymbol{x}_{k+1}, t_{k}, t_{k+1}\right)=\left(t_{R}^{j}-t_{\text {meas }}\right)-\delta t_{R}^{j}\left(t_{\text {meas }} ; \boldsymbol{x}_{k}, \boldsymbol{x}_{k+1}, t_{k}, t_{k+1}\right) \\
0=g_{2}\left(t_{\text {meas }}, t_{\text {trans }} ; \boldsymbol{x}_{k}, \boldsymbol{x}_{k+1}, t_{k}, t_{k+1}\right)=\left(t_{\text {meas }}-t_{\text {trans }}\right)-\frac{1}{c} r_{s}^{j \ell}\left(t_{\text {meas }}, t_{\text {trans }} ; \boldsymbol{x}_{k}, \boldsymbol{x}_{k+1}, t_{k}, t_{k+1}\right)
\end{gathered}
$$

where $r_{s}^{j \ell}$ is the scalar range between the vectors $\boldsymbol{r}_{s}^{j}\left(t_{\text {meas }} ; \boldsymbol{x}_{k}, \boldsymbol{x}_{k+1}, t_{k}, t_{k+1}\right)$ and $\boldsymbol{r}_{s}^{\ell}\left(t_{\text {trans }} ; \boldsymbol{x}_{k}, \boldsymbol{x}_{k+1}, t_{k}, t_{k+1}\right)$, as in the first term on the right hand side of Eq. (14). The first equation, Eq. (15a), is an implicit statement that receiver clock time $t_{R}^{j}$ equals true measurement time $t_{\text {meas }}$ plus the correction $\delta t_{R}^{j}$ that applies at $t_{\text {meas }}$. The second equation constrains the reception time $t_{\text {meas }}$ to equal $t_{\text {trans }}$ plus the light travel time between satellite $\ell$ and satellite $j$.

Note that the functions $\delta t_{R}^{j}\left(t_{\text {meas }} ; \boldsymbol{x}_{k}, \boldsymbol{x}_{k+1}, t_{k}, t_{k+1}\right), \quad \boldsymbol{r}_{s}^{j}\left(t_{\text {meas }} ; \boldsymbol{x}_{k}, \boldsymbol{x}_{k+1}, t_{k}, t_{k+1}\right), \quad$ and $\boldsymbol{r}_{s}^{\ell}\left(t_{\text {trans }} ; \boldsymbol{x}_{k}, \boldsymbol{x}_{k+1}, t_{k}, t_{k+1}\right)$ are not standard components of a typical discrete-time estimation algorithm. They assume that the estimation algorithm explicitly computes estimates of the states at sample times $t_{k}$ and $t_{k+1}$. Recall that these states are denoted by $\boldsymbol{x}_{k}$ and $\boldsymbol{x}_{k+1}$. These non-standard functions are needed to interpolate the state elements $\delta t_{R}^{j}, \boldsymbol{r}_{s}^{j}$, and $\boldsymbol{r}_{s}^{\ell}$ to the times $t_{\text {meas }}$ and $t_{\text {trans }}$, because they normally differ from $t_{k}$ and $t_{k+1}$. Any reasonable interpolation algorithm should suffice.

The pair of constraints in Eqs. (15a)-(15b) can be solved iteratively for the true times $t_{\text {meas }}$ and $t_{\text {trans }}$ by means of a Newton-Raphson approach. The effect is that $t_{\text {meas }}$ and $t_{\text {trans }}$ are implicit functions of $\boldsymbol{x}_{k}$ and $\boldsymbol{x}_{k+1}$. Substitution of these implicit functions into the right hand side of Eq. (14) yields a measurement model that effectively depends only on $\boldsymbol{x}_{k}$ and $\boldsymbol{x}_{k+1}$ :

$$
\begin{aligned}
\lambda \phi^{j \ell}=r_{s}^{j \ell}\left[t_{\text {meas }}\right. & \left.\left(\boldsymbol{x}_{k}, \boldsymbol{x}_{k+1}\right), t_{\text {trans }}\left(\boldsymbol{x}_{k}, \boldsymbol{x}_{k+1}\right) ; \boldsymbol{x}_{k}, \boldsymbol{x}_{k+1}, t_{k}, t_{k+1}\right] \\
& +c\left\{\delta t_{R}^{j}\left[t_{\text {meas }}\left(\boldsymbol{x}_{k}, \boldsymbol{x}_{k+1}\right) ; \boldsymbol{x}_{k}, \boldsymbol{x}_{k+1}, t_{k}, t_{k+1}\right]-\delta t_{R}^{\ell}\left[t_{\text {trans }}\left(\boldsymbol{x}_{k}, \boldsymbol{x}_{k+1}\right) ; \boldsymbol{x}_{k}, \boldsymbol{x}_{k+1}, t_{k}, t_{k+1}\right]\right\}+\lambda b^{j \ell} \\
& +\eta^{j \ell}
\end{aligned}
$$

Extra care must be taken not to confuse $r_{s}^{j \ell}$ with $r_{s}^{\ell j}, \phi^{j \ell}$ with $\phi^{\ell j}$, and so forth. Although they apply to the same pair of satellites, the roles of receiver and transmitter are not interchangeable in these equations.

The beat carrier phase measurement on the downlink from a satellite to a ground station is very similar to the crosslink measurement in many respects. The measurements have the same general structure, and are biased in the same way. Ground station clocks, however, are assumed to be disciplined by GPS, so that the clock error is negligible. The resulting beat carrier phase between ground station $j$ and satellite $\ell$ is

$$
\begin{gathered}
\lambda \phi_{j}^{\ell}=\sqrt{\left[\boldsymbol{r}_{g j}\left(t_{\text {meas }}\right)-\boldsymbol{r}_{s}^{\ell}\left(t_{\text {trans }} ; \boldsymbol{x}_{k}, \boldsymbol{x}_{k+1}, t_{k}, t_{k+1}\right)\right]^{\mathrm{T}}\left[\boldsymbol{r}_{g j}\left(t_{\text {meas }}\right)-\boldsymbol{r}_{s}^{\ell}\left(t_{\text {trans }} ; \boldsymbol{x}_{k}, \boldsymbol{x}_{k+1}, t_{k}, t_{k+1}\right)\right]} \\
-c \delta t_{R}^{\ell}\left[t_{\text {trans }} ; \boldsymbol{x}_{k}, \boldsymbol{x}_{k+1}, t_{k}, t_{k+1}\right]+\lambda b_{j}^{\ell}+\eta_{j}^{\ell}
\end{gathered}
$$

in which most of the quantities are defined in ways analogous to those in Eq. (14). $\boldsymbol{r}_{g j}\left(t_{\text {meas }}\right)$ is the known ground station location, which depends on time because it is given in inertial coordinates. While the true time of reception $t_{\text {meas }}$ is known here, the transmission time once again is not. A version of the second implicit constraint in Eq. (15b) must be employed to resolve this problem:

$$
0=g_{2}\left(t_{\text {trans }}\right)=\left(t_{\text {meas }}-t_{\text {trans }}\right)-\frac{1}{c} r_{j}^{\ell}\left(t_{\text {trans }} ; \boldsymbol{x}_{k}, \boldsymbol{x}_{k+1}, t_{k}, t_{k+1}\right)
$$


where $r_{j}^{\ell}\left(t_{\text {trans }} ; \boldsymbol{x}_{k}, \boldsymbol{x}_{k+1}, t_{k}, t_{k+1}\right)$ is the range that appears as the first term on the right hand side of Eq. (17).

Pseudoranges at the ground stations measure ranges to the same satellites as the corresponding beat carrier phase measurements. They do not have any unknown bias, but their noise covariance is generally much higher than the phase ranges. The pseudorange measurement equation takes the form

$$
\begin{gathered}
P_{j}^{\ell}=\sqrt{\left[\boldsymbol{r}_{g j}\left(t_{\text {meas }}\right)-\boldsymbol{r}_{s}^{\ell}\left(t_{\text {trans }} ; \boldsymbol{x}_{k}, \boldsymbol{x}_{k+1}, t_{k}, t_{k+1}\right)\right]^{\mathrm{T}}\left[\boldsymbol{r}_{g j}\left(t_{\text {meas }}\right)-\boldsymbol{r}_{s}^{\ell}\left(t_{\text {trans }} ; \boldsymbol{x}_{k}, \boldsymbol{x}_{k+1}, t_{k}, t_{k+1}\right)\right]} \\
-c \delta t_{R}^{\ell}\left[t_{\text {trans }} ; \boldsymbol{x}_{k}, \boldsymbol{x}_{k+1}, t_{k}, t_{k+1}\right]+\mu_{j}^{\ell}
\end{gathered}
$$

Once again the implicit constraint of Eq. (18) must be solved iteratively to determine the true time of transmission of the signal. The problem of implicit measurement times is common to techniques that measure ranges by calculating transmission delay. Readers interested in this topic are encouraged to refer to ${ }^{29}$.

No terms are included for the ionosphere or neutral atmosphere in the carrier phase and pseudorange measurement models, and this omission is intentional. It is assumed that these components can be modeled well enough to be corrected and removed from the ground-based measurements. Work is ongoing in this area. It includes efforts to use dual-frequency GPS measurements of TEC, available at ground stations, to create a local model of the ionosphere ${ }^{30}$.

Each of the three measurement models takes the generic form

$$
y_{k+1}=h\left(k ; \boldsymbol{x}_{k}, \boldsymbol{x}_{k+1}\right)+v_{k+1}
$$

where $v_{k+1}$ is the scalar error for this measurement. This equation presumes solution for the implicitly defined times $t_{\text {meas }}$ and $t_{\text {trans }}$ and substitution of these solutions into the measurement models as in Eq. (16) for the crosslinks case. A carrier phase version of Eq. (16) can be developed from Eq. (17) and a pseudorange version from Eq. (19).

Typically, multiple measurements will occur in the sample interval $\left[t_{k}, t_{k+1}\right)$. That is, $t_{k} \leq t_{\text {meas }}<t_{k+1}$ and $t_{k} \leq t_{\text {trans }}<t_{k+1}$ for each such measurement. (The rare measurements that overlap two sample intervals can be discarded for the sake of simplicity.) All these measurements and their corresponding model functions can be stacked to form the vector measurement equation

$$
\boldsymbol{y}_{k+1}=h\left(k ; \boldsymbol{x}_{k}, \boldsymbol{x}_{k+1}\right)+\boldsymbol{v}_{k+1}
$$

This is the general, but non-standard, form that will be used in this paper's observability analysis.

\section{Derivation of the Observability Analysis Equations}

Conceptually, a system is observable when it is possible to uniquely determine the initial state of the system from some finite number of measurements. In the field of estimation, it is often useful to know whether a given system is observable before making a possibly futile attempt to estimate its state. That is the main idea behind this paper: to study whether it is theoretically possible to determine atmospheric density and the orbits of a satellite constellation, given the proposed measurements. A closely related concept is constructability, which asks whether the measurements are sufficient to uniquely determine the final state, as opposed to initial state. The conditions are mathematically equivalent in cases, including the current case, where the system dynamics are invertible. For this reason this paper will continue to use the terminology observability analysis, although technically it performs a constructability analysis due to constructability's closer connection with the standard filtering problem.

If a system is linear, then it either is or is not observable. For nonlinear systems, however, it may not be possible to make such a clear distinction. Asking whether a system is observable is equivalent to asking whether a cost function based on measurement error residuals has a global minimum. Many nonlinear systems have a distinct global minimum, but may also have additional distinct local minima. This paper examines the local uniqueness, i.e. the isolation, of the global minimum at the true state. It does not address the question of whether other distinct local minima exist. Local uniqueness is analyzed via linearization about the true state in a manner analogous to an extended Kalman filter (EKF). This type of analysis is called a linearized observability analysis. 
The observability analysis linearizes the dynamics and measurement models of Eqs. (6) and (21) in Section III about a nominal state time history $\boldsymbol{x}_{0}, \boldsymbol{x}_{1}, \boldsymbol{x}_{2}, \ldots, \boldsymbol{x}_{N}$. The nominal process noise is zero, since $\boldsymbol{w}_{k}$ is modeled as a zero-mean random process. The linearized dynamics and measurement models are

$$
\begin{gathered}
\Delta \boldsymbol{x}_{k+1}=\Phi_{k} \Delta \boldsymbol{x}_{k}+\Gamma_{k} \boldsymbol{w}_{k} \\
\Delta \boldsymbol{y}_{k+1}=\widehat{H}_{k} \Delta \boldsymbol{x}_{k}+\bar{H}_{k+1} \Delta \boldsymbol{x}_{k+1}+\boldsymbol{v}_{k+1}
\end{gathered}
$$

where $\Phi_{k}=\left.\frac{\partial f}{\partial x}\right|_{k, x_{k}, 0}$ and $\Gamma_{k}=\left.\frac{\partial f}{\partial \boldsymbol{w}}\right|_{k, x_{k}, 0}$ in the dynamics equation. Likewise, $\widehat{H}_{k}=\left.\frac{\partial \boldsymbol{h}}{\partial \boldsymbol{x}_{k}}\right|_{k, \boldsymbol{x}_{k}, \boldsymbol{x}_{k+1}, 0}$ and $\bar{H}_{k+1}=$ $\left.\frac{\partial \boldsymbol{h}}{\partial \boldsymbol{x}_{k+1}}\right|_{k, \boldsymbol{x}_{k}, \boldsymbol{x}_{k+1}, 0}$ in the measurement equation. The quantity $\Delta \boldsymbol{x}_{k}$ is the state perturbation from the nominal linearizing value $\boldsymbol{x}_{k}$, and $\Delta \boldsymbol{y}_{k+1}$ is the difference between $\boldsymbol{y}_{k+1}$ and $\boldsymbol{h}$ evaluated at the linearizing values $\boldsymbol{x}_{k}$ and $\boldsymbol{x}_{k+1}$.

To gain insight into the observability analysis, consider the simple linear case

$$
\boldsymbol{y}=H \boldsymbol{x}
$$

The well-known least squares solution for $\boldsymbol{x}$ in this equation is given by

$$
\boldsymbol{x}=\left(H^{\mathrm{T}} H\right)^{-1} H^{\mathrm{T}} \boldsymbol{y}
$$

In this equation, the requirement to be able to determine $x$ uniquely is that the matrix $H^{\mathrm{T}} H$ be invertible. For that to hold, $H$ must have at least as many rows as columns (i.e. enough measurements) and it must be full-rank. Transferring these ideas to a typical linear dynamic system, the system dynamics can be used to express the state at any time $k$ as a linear function of the state at any other time, if one assumes zero process noise. Using the dynamics in this way, a whole sequence of measurements at different times can all be referred to the initial or final state, and this batch of measurements can be stacked together to form a linearized measurement model that looks like Eq. (24).

$$
\left[\begin{array}{c}
\boldsymbol{y}_{1} \\
\boldsymbol{y}_{2} \\
\vdots \\
\boldsymbol{y}_{N}
\end{array}\right]=\left[\begin{array}{c}
H_{1} \\
H_{2} \\
\vdots \\
H_{N}
\end{array}\right] \boldsymbol{x}_{N}, \text { or equivalently, } \boldsymbol{y}_{\text {batch }}=\mathcal{H} \boldsymbol{x}_{N}
$$

where $\boldsymbol{y}_{\text {batch }}=\left[\boldsymbol{y}_{1} ; \boldsymbol{y}_{2} ; \ldots ; \boldsymbol{y}_{N}\right]$ and where $\mathcal{H}$ is the block matrix in the middle expression of Eq. (26). By analogy with the simpler equation, the system is observable if the large batch matrix $\mathcal{H}^{\mathrm{T}} \mathcal{H}$ is invertible. Note that $\mathcal{H}^{\mathrm{T}} \mathcal{H}$ is the constructability Gramian of the linearized system. This is the most basic form of batch observability analysis, which neglects the effects of process and measurement noise. It does not yet explicitly consider a measurement equation that depends on the previous state as well as the current state as in Eq. (23). If the error in $\boldsymbol{y}_{\text {batch }}$ has been normalized to have identity covariance, then estimation theory demonstrates that the matrix $\left(\mathcal{H}^{\mathrm{T}} \mathcal{H}\right)^{-1}$ is equivalent to the covariance matrix $P_{x x N}$ for the state estimation error after $N$ measurements.

Another observability criterion is equivalent to the criterion that $\mathcal{H}^{\mathrm{T}} \mathcal{H}$ be invertible: All diagonal elements of $\left(\mathcal{H}^{\mathrm{T}} \mathcal{H}\right)^{-1}$ must be finite. The numerical observability tests used in this paper are based on computing $\left(\mathcal{H}^{\mathrm{T}} \mathcal{H}\right)^{-1}$. If the inversion fails, then the system is not observable. If it succeeds, the system may still be unobservable, in which case the successful inversion would be the result of round-off error. Alternatively, the system may be observable, but only weakly so. In either case, one or more of the diagonal elements of $\left(\mathcal{H}^{\mathrm{T}} \mathcal{H}\right)^{-1}$ will be very large, indicating a large estimation error variance for the corresponding state. In summary, this paper's observability test requires that $\mathcal{H}^{\mathrm{T}} \mathcal{H}$ be numerically invertible and that all diagonal elements of $\left(\mathcal{H}^{\mathrm{T}} \mathcal{H}\right)^{-1}$ be sufficiently small, as in Ref. 25. 
After significant algebraic manipulation of Eqs. (22) and (23), an equivalent batch measurement equation for the associated Kalman filter can be shown to be

$$
\begin{aligned}
{\left[\begin{array}{c}
\Delta \boldsymbol{y}_{1} \\
\Delta \boldsymbol{y}_{2} \\
\vdots \\
\Delta \boldsymbol{y}_{N}
\end{array}\right] } & =\left[\begin{array}{c}
\widehat{H}_{0} \widetilde{\Phi}_{N, 0}^{-1}+\bar{H}_{1} \widetilde{\Phi}_{N, 1}^{-1} \\
\widehat{H}_{1} \widetilde{\Phi}_{N, 1}^{-1}+\bar{H}_{2} \widetilde{\Phi}_{N, 2}^{-1} \\
\vdots \\
\widehat{H}_{N-1} \widetilde{\Phi}_{N, N-1}^{-1}+\bar{H}_{N}
\end{array}\right] \Delta \boldsymbol{x}_{N} \\
& -\left[\begin{array}{cccc}
\widehat{H}_{0} \widetilde{\Phi}_{1,0}^{-1} \Gamma_{0} & \left(\widehat{H}_{0} \widetilde{\Phi}_{2,0}^{-1}+\bar{H}_{1} \widetilde{\Phi}_{2,1}^{-1}\right) \Gamma_{1} & \cdots & \left(\widehat{H}_{0} \widetilde{\Phi}_{N, 0}^{-1}+\bar{H}_{1} \widetilde{\Phi}_{N, 1}^{-1}\right) \Gamma_{N-1} \\
0 & \widehat{H}_{1} \widetilde{\Phi}_{2,1}^{-1} \Gamma_{1} & \cdots & \left(\widehat{H}_{1} \widetilde{\Phi}_{N, 1}^{-1}+\bar{H}_{2} \widetilde{\Phi}_{N, 2}^{-1}\right) \Gamma_{N-1} \\
\vdots & \vdots & \ddots & \vdots \\
0 & 0 & \cdots & \widehat{H}_{N-1} \widetilde{\Phi}_{N, N-1}^{-1} \Gamma_{N-1}
\end{array}\right]\left[\begin{array}{c}
\boldsymbol{w}_{0} \\
\boldsymbol{w}_{1} \\
\vdots \\
\boldsymbol{w}_{N-1}
\end{array}\right]+\left[\begin{array}{c}
\boldsymbol{v}_{1} \\
\boldsymbol{v}_{2} \\
\vdots \\
\boldsymbol{v}_{N}
\end{array}\right]
\end{aligned}
$$

where the inverse state transition matrices $\widetilde{\Phi}_{k, j}^{-1}$ are defined recursively as follows:

$$
\begin{gathered}
\widetilde{\Phi}_{k, j}^{-1}=\left(\widetilde{\Phi}_{k, j}\right)^{-1} \\
\widetilde{\Phi}_{k, j}=\Phi_{k-1} \widetilde{\Phi}_{k-1, j}, \widetilde{\Phi}_{j, j}=I
\end{gathered}
$$

The batch equation can be further simplified by defining some names for the large batch matrices and vectors:

$$
\Delta \boldsymbol{y}_{\text {batch }}=\mathcal{H} \Delta \boldsymbol{x}_{N}+\left(\mathcal{G} \boldsymbol{w}_{\text {batch }}+\boldsymbol{v}_{\text {batch }}\right)
$$

where $\Delta \boldsymbol{y}_{\text {batch }}=\left[\Delta \boldsymbol{y}_{1} ; \Delta \boldsymbol{y}_{2} ; \ldots ; \Delta \boldsymbol{y}_{N}\right], \boldsymbol{w}_{\text {batch }}=\left[\boldsymbol{w}_{0} ; \boldsymbol{w}_{1} ; \ldots ; \boldsymbol{w}_{N-1}\right], \boldsymbol{v}_{\text {batch }}=\left[\boldsymbol{v}_{1} ; \boldsymbol{v}_{2} ; \ldots ; \boldsymbol{v}_{N}\right]$, and where $\mathcal{H}$ and $\mathcal{G}$ are the two block matrices on the right hand side of Eq. (27). As this equation shows, the quantity ( $\mathcal{G} \boldsymbol{w}_{\text {batch }}+$ $\boldsymbol{v}_{\text {batch }}$ ), which contains both process and measurement noise, acts collectively as noise for the batch measurement equation. If $\mathcal{R}_{\text {batch }}$ is the covariance of the measurement noise time history $\boldsymbol{v}_{\text {batch }}$ and $\mathcal{Q}_{\text {batch }}$ is the covariance of the process noise time history $\boldsymbol{w}_{\text {batch }}$, then, assuming they are uncorrelated, the combined covariance is given by

$$
\mathrm{E}\left[\left(\mathcal{G} \boldsymbol{w}_{\text {batch }}+\boldsymbol{v}_{\text {batch }}\right)\left(\mathcal{G} \boldsymbol{w}_{\text {batch }}+\boldsymbol{v}_{\text {batch }}\right)^{\mathrm{T}}\right]=\mathcal{G} \mathcal{Q}_{\text {batch }} \mathcal{G}^{\mathrm{T}}+\mathcal{R}_{\text {batch }}
$$

It is common in batch observability calculations to weight the measurement errors, or equivalently, to transform the batch measurements so that the transformed measurement error has identity covariance. This practice has the effect of placing more importance on a measurement if it is known to be more accurate and disregarding to some extent those measurements that are very noisy. Implementation via transformation results in a transformed $\mathcal{H}$ matrix. Let it be called $\widetilde{\mathcal{H}}$. The use of $\widetilde{\mathcal{H}}$ in numerical observability calculations yields a covariance $\left(\widetilde{\mathcal{H}}^{\mathrm{T}} \widetilde{\mathcal{H}}\right)^{-1}$ that closely reflects the actual amount of measurement information about each state. The diagonal elements of $\left(\widetilde{\mathcal{H}}^{\mathrm{T}} \widetilde{\mathcal{H}}\right)^{-1}$ give reasonable measures of the relative observability of the corresponding states. To perform this transformation, let

$$
\tilde{R}_{\text {batch }}^{-1} \tilde{R}_{\text {batch }}^{-\mathrm{T}}=\left(\mathcal{G} \mathcal{Q}_{\text {batch }} \mathcal{G}^{\mathrm{T}}+\mathcal{R}_{\text {batch }}\right)
$$

that is, $\tilde{R}_{\text {batch }}$ is the square-root information matrix for the random vector $\left(\mathcal{G} \boldsymbol{w}_{\text {batch }}+\boldsymbol{v}_{\text {batch }}\right)$. Then define

$$
\begin{gathered}
\tilde{\boldsymbol{v}}_{\text {batch }}=\tilde{R}_{\text {batch }}\left(\mathcal{G} \boldsymbol{w}_{\text {batch }}+\boldsymbol{v}_{\text {batch }}\right) \\
\Delta \widetilde{\boldsymbol{y}}_{\text {batch }}=\widetilde{R}_{\text {batch }} \Delta \boldsymbol{y}_{\text {batch }} \\
\widetilde{\mathcal{H}}=\widetilde{R}_{\text {batch }} \mathcal{H} \mathcal{H}
\end{gathered}
$$


The transformed batch equation

$$
\Delta \widetilde{\boldsymbol{y}}_{\text {batch }}=\widetilde{\mathcal{H}} \Delta \boldsymbol{x}_{N}+\widetilde{\boldsymbol{v}}_{\text {batch }}
$$

has identity error covariance. The system is considered observable if $\widetilde{\mathcal{H}}^{\mathrm{T}} \widetilde{\mathcal{H}}$ is invertible. In fact, the observability of $\Delta \boldsymbol{x}_{N}$ in Eq. (29) is equivalent to its observability in Eq. (33) because $\widetilde{R}_{\text {batch }}$ in the last line of Eq. (32) is invertible. Thus the inclusion of process and measurement noise in the batch observability analysis has no effect on the theoretical observability of the system. It only influences the relative observability in a way that adds realism.

The batch observability analysis presented in Eq. (33) above is equivalent to a properly formulated square root information filter (SRIF) that performs only the covariance part of the filtering calculations. Rather than form the unwieldy batch matrix $\widetilde{\mathcal{H}}$, the recursive SRIF directly tracks the inverse square root of the state vector covariance and deals with process noise automatically. It also more easily handles the changing length of the state vector introduced by the measurement bias states.

For a linear system, it is possible to completely decouple the covariance portion of the SRIF from the state estimation. This paper's local observability analysis, however, requires linearization about accurate states. The need to compute state estimates is avoided by linearizing about truth-model states. A local observability analysis only answers the question of solution uniqueness near the true states. Linearization about the true states rather than nearby estimates thus provides the proper measure of local observability. One must take care to choose realistic initial conditions for the truth model, and typically one considers observability under multiple scenarios as dictated by differing initial truth states.

A traditional SRIF, like a Kalman filter, proceeds in two stages: dynamic propagation first, and then measurement update ${ }^{31}$. The two-stage filtering procedure is somewhat complicated by a measurement model like that in Eq. (23) that depends not only on the current state, but also on the previous state. In this situation, it makes more sense to derive a form of the SRIF that performs the dynamic propagation and measurement update steps simultaneously, as in Ref. 32.

The SRIF effectively stores both the state estimate and the state estimation error covariance in a square-root information equation of the form

$$
\hat{R}_{x x k}\left(\Delta \boldsymbol{x}_{k}-\Delta \widehat{\boldsymbol{x}}_{k}\right)=-\hat{\boldsymbol{v}}_{x k}
$$

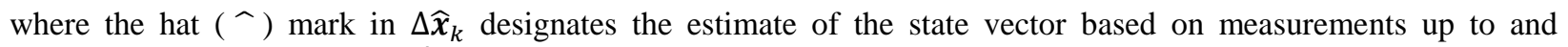
including time $k$, the matrix $\hat{R}_{x x k}$ is the square-root information matrix for the state, and the error between the estimate and the true state is characterized by the zero-mean, identity-covariance white noise sequence $\hat{\boldsymbol{v}}_{x k} . \hat{R}_{x x k}$ is related to the state's estimation error covariance matrix according to $\hat{P}_{x x k}=\hat{R}_{x x k}^{-1} \hat{R}_{x x k}^{-\mathrm{T}}$. For this form of the SRIF, the simultaneous dynamic propagation and measurement update is given by

$$
Q_{k+1}\left[\begin{array}{cc}
\hat{R}_{w w k} & \hat{R}_{w x k} \\
0 & \hat{R}_{x x k+1} \\
0 & 0
\end{array}\right]=\left[\begin{array}{cc}
R_{w w k} & 0 \\
-\hat{R}_{x x k} \Phi_{k}^{-1} \Gamma_{k} & \hat{R}_{x x k} \Phi_{k}^{-1} \\
-R_{v v k} \widehat{H}_{k} \Phi_{k}^{-1} \Gamma_{k} & R_{v v k}\left(\widehat{H}_{k} \Phi_{k}^{-1}+\bar{H}_{k+1}\right)
\end{array}\right]
$$

In this equation, the matrices $\Phi_{k}, \Gamma_{k}, \widehat{H}_{k}$, and $\bar{H}_{k+1}$ are taken from the system's linearized dynamics and measurement models given by Eqs. (22) and (23). The matrices $R_{w w k}$ and $R_{v v k}$ are the inverse-square-roots of the covariances of the process noise $\boldsymbol{w}_{k}$ and measurement noise $\boldsymbol{v}_{k}$, respectively. The calculation proceeds by $\mathrm{QR}$ factorization of the matrix on the right. One of its outputs is the matrix $\hat{R}_{x x k+1}$, which is the square-root information matrix for the error in the new state estimate $\Delta \widehat{\boldsymbol{x}}_{k+1}$. Note, however, that the new state estimate need never be formed, provided values for linearization are available from a truth model simulation. Observability after $N$ measurements is determined by the matrix $\hat{R}_{x x N}$, which must be full-rank. Equivalently, $\hat{R}_{x x N}$ must be numerically invertible and the covariance $\hat{P}_{x x N}=\hat{R}_{x x N}^{-1} \hat{R}_{x x N}^{-\mathrm{T}}$ must have finite values on its diagonal, signifying a finite amount of uncertainty in each of the state estimates.

The matrix $\hat{P}_{x x N}$ is equivalent to $\left(\widetilde{\mathcal{H}}^{\mathrm{T}} \widetilde{\mathcal{H}}\right)^{-1}$ associated with Eq. (33) if and only if the SRIF calculations are initiated with $\hat{R}_{x x 0}=0$. This is the condition of 0 a priori information, consistent with the question of system observability based on measurement data alone. 
If the system is unobservable, it may be reasonable to relax slightly the assumption that $\hat{R}_{x x 0}=0$. Typically, one has some a priori information, such as a bound on the physically realistic range of inverse ballistic coefficients. Such information can be incorporated into $\hat{R}_{x x 0}$ and may make the system practically observable.

\section{Results}

The observability analysis was performed in several stages. First, a truth model was used to generate several representative system state histories. Next, the dynamics and measurement models of Eqs. (6) and (21) were linearized around these "truth" states as in Eqs. (22)-(23). The SRIF covariance calculations yielded $\hat{R}_{x x N}$, the square-root information matrix for the state after $N$ measurements. Finally, $\hat{R}_{x x N}$ and the estimation error covariance $\hat{P}_{x x N}$ were examined to determine observability.

\section{A. Truth model cases}

The truth model for a representative case used for this paper's analysis includes 66 satellites in 6 nearly polar orbital planes. Each satellite has a near-circular orbit, and the mean orbit altitude for all satellites is $790 \mathrm{~km}$. The satellites are modeled as each having approximately the same truth-cross-sectional area and mass, such that the mean ratios are $C_{D} \frac{A_{v 0}}{m_{s}}=0.0134 \mathrm{~m}^{2} / \mathrm{kg}$ and $C_{S} \frac{A_{s 0}}{m_{s}}=0.0196 \mathrm{~m}^{2} / \mathrm{kg}$, and the inverse ballistic coefficient $\beta_{D}^{j}$ and solar radiation pressure coefficient $\beta_{S}^{j}$ are slowly-varying random walks that are essentially constants over the batch lengths considered in this paper. The satellite clocks are assumed to have noise characterized by

$$
\begin{gathered}
\mathrm{E}\left[w_{\phi}^{j}(t) w_{\phi}^{j}(\tau)\right]=\left(1.6 \times 10^{-22} \mathrm{sec}\right) \delta(t-\tau) \\
\mathrm{E}\left[w_{f}^{j}(t) w_{f}^{j}(\tau)\right]=\left(2.5 \times 10^{-26} / \mathrm{sec}\right) \delta(t-\tau) \\
\mathrm{E}\left[w_{\phi}^{j}(t) w_{f}^{j}(\tau)\right]=0
\end{gathered}
$$

These process noise intensities correspond to an equivalent root Allan variance of $1.5 \times 10^{-12}$ at a delay of $139 \mathrm{sec}$, typical of a good oven-controlled crystal oscillator (OCXO) ${ }^{26}$ under benign conditions.

The satellites are tracked by 12 ground reference stations. Each ground station also receives GPS signals that are used to discipline the ground station clocks. The ground stations are located as shown in the map in Figure 2.

Within each orbital plane, adjacent satellites are connected by crosslink measurements, and every ground station has phase and pseudorange downlink measurements from all satellites above an elevation of $10^{\circ}$. For simplicity, all the crosslink and downlink measurements have sampling rates of 5 seconds. The crosslink accumulated delta range measurements are

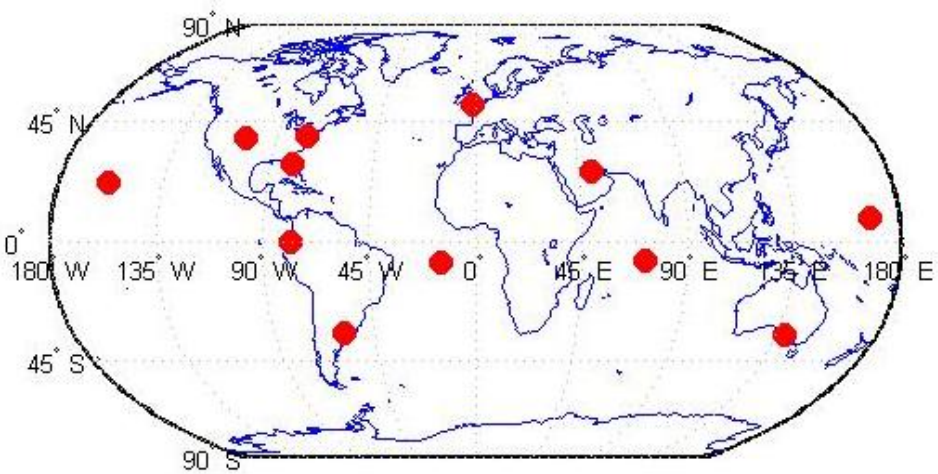

Figure 2. Map of ground reference stations modeled as having measurement error standard deviations of $3.4 \mathrm{~cm}$. On the accumulated delta range downlink measurements, the measurement error standard deviations are $0.3 \mathrm{~mm}$. In contrast, the unbiased but imprecise pseudoranges have measurement error standard deviations of $30 \mathrm{~m}$. Note: These accuracies assume averaging of less accurate, higher frequency data over 5 -second intervals.

The "truth" splined atmospheric density distribution is initialized by fitting to the outputs of the NRLMSISE-00 model evaluated for January 21 at 08:03:20 UTC, with $F_{10.7}=150$, the 81-day average $\overline{F_{10.7}}=150$, and $a_{p}=4$. The spline has 6 longitude nodes and 5 latitude nodes, including the poles. Markov time constants of $\tau_{a i}=3$ hours were selected based on the maximum available time resolution of the geomagnetic and solar indices, which in turn limits 
the resolution of standard models that use these indices as inputs. The intensity of the driving process noise $w_{a i}$ is known to be related to the steady-state variance of $p_{a i}$ according to

$$
\mathrm{E}\left[w_{a i}(t) w_{a i}(\tau)\right]=\frac{2}{\tau_{a i}} \sigma_{a i}^{2} \delta(t-\tau)
$$

where $\sigma_{a i}^{2}$, the steady-state variance of $p_{a i}$, is chosen to yield an equivalent density steady-state standard deviation of approximately $70 \%$. For simplicity, the elements $w_{a i}$ and $w_{a j}$ are assumed to be uncorrelated for $i \neq j$.

The truth model simulation was performed for a batch length of 12 hours. The samples all had equal lengths of 5 seconds.

Other truth model cases for which observability has been analyzed are similar to this representative case in many respects. They attempt to isolate the effects on observability of certain types of changes. For instance, one doubles the batch length to 24 hours. Another alternate case uses the lower constellation altitude of $500 \mathrm{~km}$.

Of course one could think of many other interesting cases. These might include changes of atmospheric Markov time constants $\tau_{a i}$ or process noise intensities to explore performance under conditions of high solar activity, changes of measurement precision, changes in the set of ground reference stations, or changes to the constellation configuration. For the sake of brevity, a total of only three cases are considered here.

\section{B. Representative observability results}

The observability analysis results presented here are for the representative case described in the previous subsection. In this instance, the squareroot information matrix $\hat{R}_{x x N}$ was fullrank, as demonstrated by the success of the numerical inversion $\hat{R}_{x x N}^{-1} \hat{R}_{x x N}^{-\mathrm{T}}$. The system was still deemed unobservable, however, because the diagonal elements of $\hat{P}_{x x N}$ corresponding to the atmospheric parameter vector $\boldsymbol{p}_{a}$ all had very large values, orders of magnitude above the parameter values themselves. Closer examination revealed that these standard deviations were decreasing and had not yet reached a steady state at the end of the 12 hour batch, as shown in Figure 3. For this representative case, the squareroot information matrix $\hat{R}_{x x 0}$ contained no a priori information about the atmospheric density parameters or the

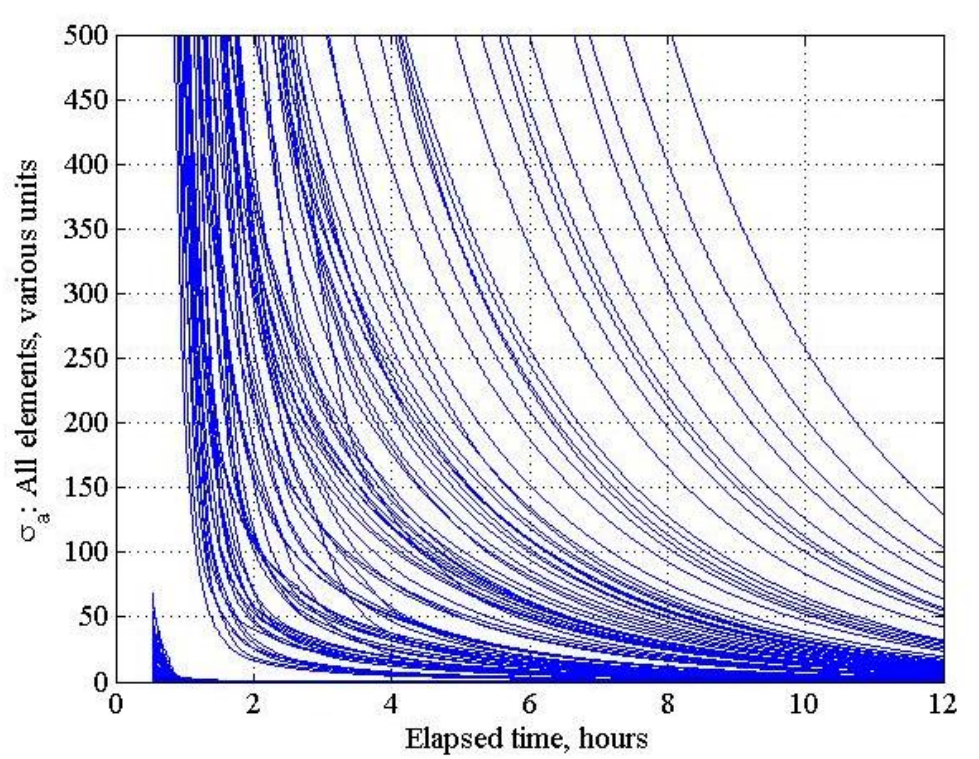

Figure 3. Estimation error standard deviations for the elements of $p_{a}$, representative case

inverse ballistic coefficients, so effectively the initial covariance was infinite. The finite standard deviations shown in Figure 3 mean that the crosslink and downlink measurements did improve the estimates of those parameters. The measurements were not sufficient, however, to achieve trustworthy estimates within a reasonable amount of time, and so this particular case can be considered practically unobservable.

Despite the large remaining uncertainties associated with $\boldsymbol{p}_{a}$, the satellite orbit accuracies were reasonable for this representative case, as were those of the inverse ballistic coefficient, solar radiation pressure coefficient, and other satellite-specific states. Figure 4 shows the estimation error standard deviations for one satellite in the altitude/along-track/cross-track directions, and Figure 5 likewise gives the estimation error standard deviations for the same satellite's drag and solar pressure coefficients. The high level of orbital position accuracy in Figure 4 is somewhat surprising, given the poor accuracy of the atmospheric parameters. These results suggest that this is a data-rich orbit estimation problem, in which the final accuracy is insensitive to significant levels of dynamic model error. It is conjectured that the crosslinks are the key to this data richness. 


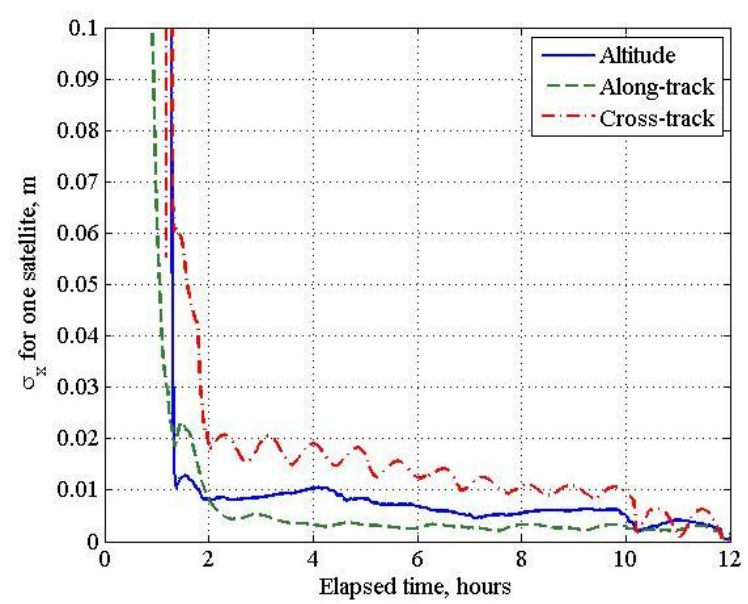

Figure 4. Estimation error standard deviations for one satellite's position (in the altitude/along-track/crosstrack directions)

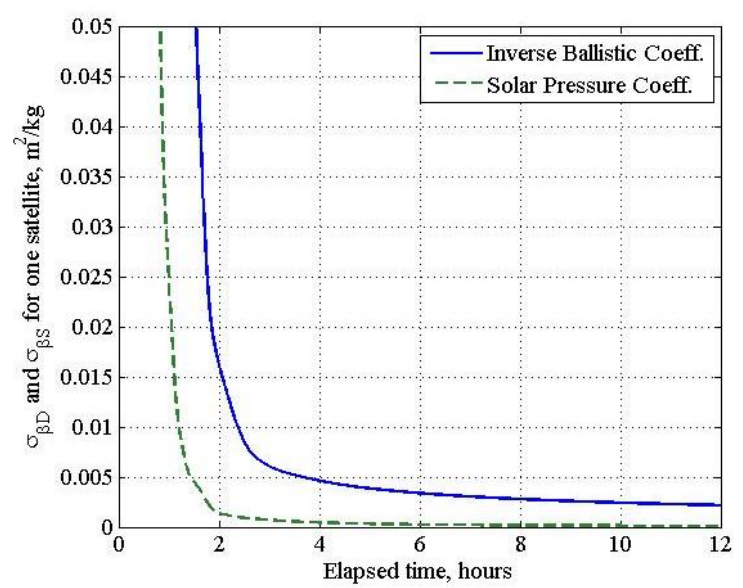

Figure 5. Estimation error standard deviations for one satellite's inverse ballistic coefficient and solar radiation pressure coefficient
In an attempt to overcome the original unobservability of the system, a priori knowledge was incorporated by setting some of the elements in $\hat{R}_{x x 0}$ to non-zero values. To begin with, the elements corresponding to inverse ballistic coefficients $\beta_{D}^{j}$ were adjusted to reflect a physically realistic level of initial uncertainty about these values for wellcharacterized satellites. Most notably, this had the effect of reducing the uncertainty about inverse ballistic coefficients throughout the entire 12-hour batch, as illustrated for one satellite in Figure 6. The diagonal elements of $\hat{P}_{x x k}$ corresponding to the satellite orbit states and the atmospheric parameters initially decreased more quickly, but after three or four hours the standard deviations returned to those of the first representative case. This transient effect is shown in Figure 7 and Figure 8. Such behavior suggests that errors in the estimates of inverse ballistic coefficients are not a major contributor to errors in $\boldsymbol{p}_{a}$ estimates, at least in the long term.

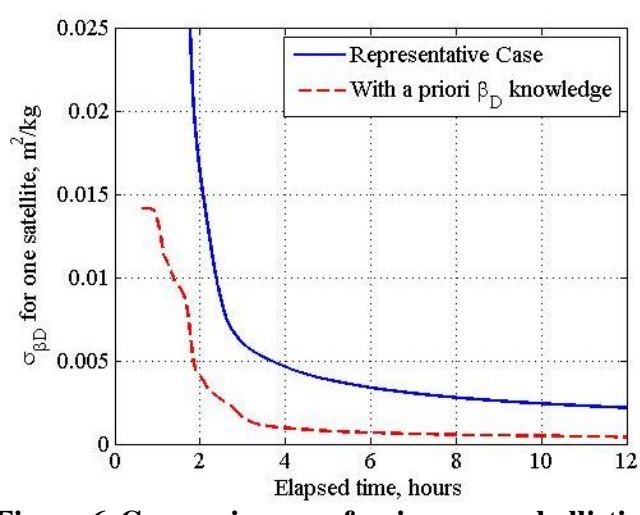

Figure 6. Comparison of inverse ballistic coefficient estimation error standard deviations, with and without a priori knowledge included

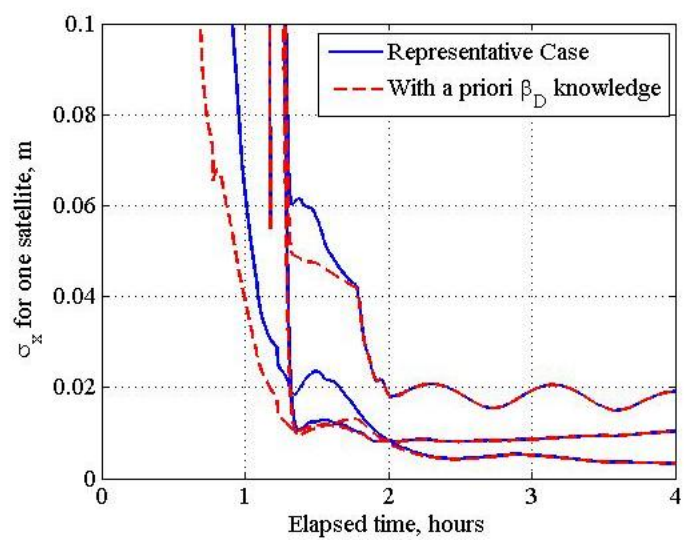

Figure 7. Comparison of position estimation error standard deviations for one satellite, showing transient behavior

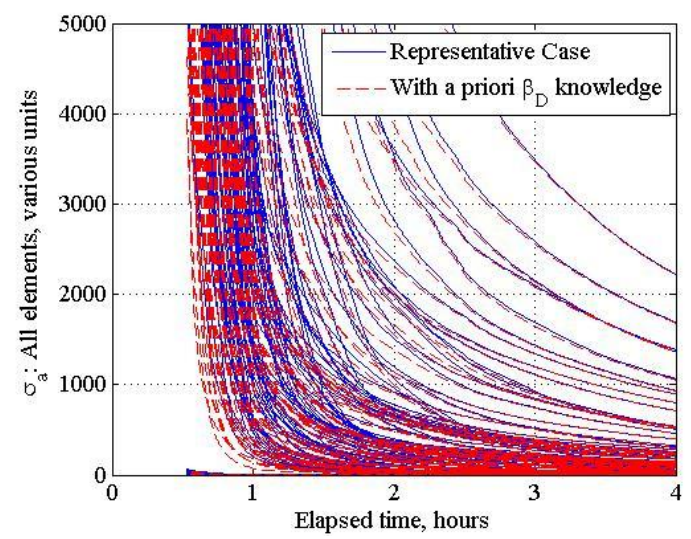

Figure 8. Comparison of atmospheric parameters estimation error standard deviations, showing transient behavior 
Of course, changes in the Markov time constants of the atmospheric states could affect this lack of coupling, especially if the time constants increased dramatically. Such an increase would yield a random-walk-like model.

Further observability improvements were attempted by incorporating some reasonable $a$ priori information about the atmospheric density parameter vector $\boldsymbol{p}_{a}$, consistent with the amount of knowledge available from existing atmospheric density models. This was accomplished by setting the corresponding diagonal elements of $\hat{R}_{x x 0}$ to the inverse of the chosen Markov process standard deviations. At steady state with no incoming measurements, this is the amount of uncertainty that would be expected in $\boldsymbol{p}_{a}$ with the given dynamic model. Consequently, any reduction in the diagonal elements of $\hat{R}_{x x N}$ indicates that the atmospheric parameters are observable and the measurements are reducing the uncertainty associated with the density estimates.

The uncertainty in the elements of $\boldsymbol{p}_{a}$ does indeed decrease from its initial level over the batch interval, as shown in Figure 9. Several things can be observed in this figure. First, the elements of the parameter vector $\boldsymbol{p}_{a}$ span several orders of magnitude and are not all measured in the same units, so they cannot all be clearly visualized on one plot. The horizontal lines in the lower part of the Figure 9 are actually decreasing standard deviations corresponding to lower-magnitude elements, but the scale obscures the reduction in uncertainty. Next, while some standard deviations on this plot decrease rapidly, others are only mildly observable and the reduction is slight. One significant difference between Figure 9 and Figure 3 from the baseline representative case, other than the plot scale,

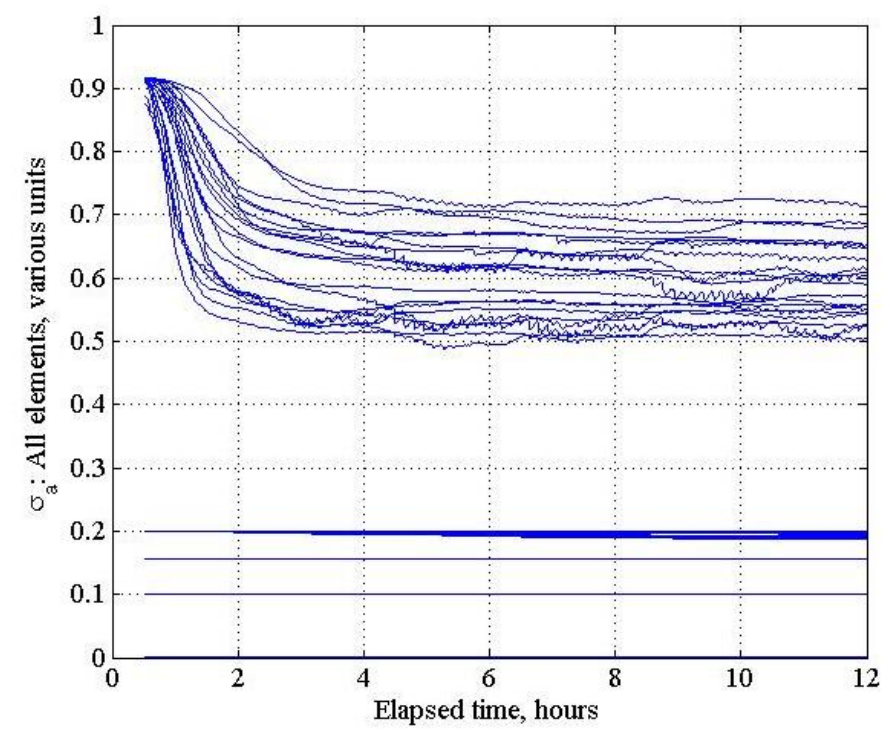

Figure 9. Estimation error standard deviations for elements of $\boldsymbol{p}_{\boldsymbol{a}}$, with a priori density information is the way in which many of the standard deviations in this figure appear to reach a steady-state value before the end of the interval. These steady-state standard deviations suggest that additional measurements beyond this batch will not greatly improve the density estimates. A final interesting feature of this plot is the high-frequency oscillation evident in many of the standard deviation elements at equilibrium. Its period of about 8-10 minutes suggests that it may be related to the time interval between flybys of two consecutive satellites at a given location; the passage of a satellite could temporarily improve local density information.

Interpreting the results of a plot like Figure 9 is clearly challenging, particularly with so many different units and orders of magnitude represented in $\boldsymbol{p}_{a}$. After all, the real quantity of interest is density itself, and not some set of spline parameters with limited physical meaning. To this end, a method is desired to calculate the standard deviation of the density estimation error for an arbitrary position in the constellation. Such a method is readily available by using the partial derivative $\frac{\partial \rho}{\partial \boldsymbol{p}_{a}}$ to transform the covariance of the $\boldsymbol{p}_{a}$ estimates into standard deviations of density $\rho$. The transformation takes the form

$$
\sigma_{\rho}=\sqrt{\left(\frac{\partial \rho}{\partial \boldsymbol{p}_{a}}\right) P_{\boldsymbol{p}_{a} \boldsymbol{p}_{a}\left(\frac{\partial \rho}{\partial \boldsymbol{p}_{a}}\right)^{\mathrm{T}}}}
$$

where $\sigma_{\rho}$ is the density estimation error standard deviation for the location at which the derivative $\frac{\partial \rho}{\partial \boldsymbol{p}_{a}}$ was evaluated, and $P_{\boldsymbol{p}_{a} \boldsymbol{p}_{a}}$ is the block of the covariance matrix $\hat{P}_{x x N}$ corresponding to the elements of $\boldsymbol{p}_{a}$. Equation (38) has been applied to a number of random globally distributed points to determine the overall effect of the measurements on the density estimates when a priori information about $\boldsymbol{p}_{a}$ has been employed. Averaged over 1000 points in the constellation region, the normalized standard deviation $\sigma_{\rho} / \rho$ at the beginning of the batch interval was 0.71 . At the end of 12 hours, the average $\sigma_{\rho} / \rho$ was 0.44 , indicating a significant uncertainty reduction. For a better visualization of this overall drop in uncertainty, see Figure 10 and Figure 11, which have been plotted on the same scale for easy comparison.

17

American Institute of Aeronautics and Astronautics 


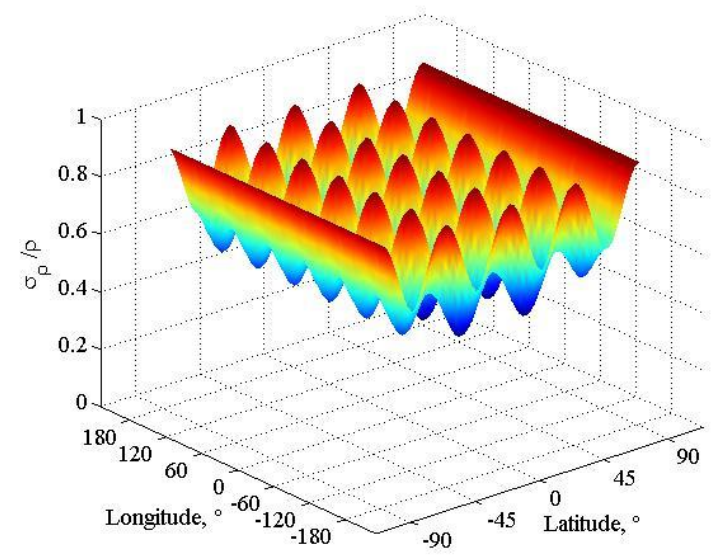

Figure 10. Normalized standard deviation of density estimate before measurements

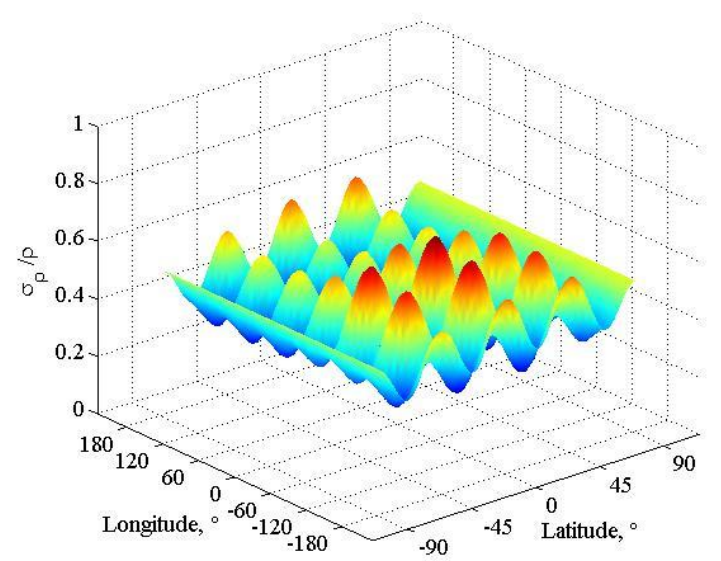

Figure 11. Normalized standard deviation of density estimate after 12-hour batch interval

In these figures, normalized density standard deviation is plotted versus latitude and longitude. The regular, bumpy pattern is closely aligned with the grid of points underlying the spline structure of the atmospheric density representation. It is at least in part an artifact of non-optimal relative scaling of $\sigma_{a i}$, the chosen steady-state Markov process standard deviations of the individual elements of $\boldsymbol{p}_{a}$. It is difficult to scale the initial uncertainties of bicubic spline parameters in a way that would produce a perfectly flat plot in Figure 10. The figures nevertheless clearly show an overall reduction in uncertainty level over the course of the 12-hour batch interval.

\section{Other scenarios}

When the batch length was doubled relative to the representative case, it had only minor effects on the results. In general, the main difference was that some of the estimation error standard deviations that had not completely reached a steady state in 12 hours were able to do so in 24 hours. For instance, Figure 12 shows the evolution of the position estimation error standard deviations for one satellite over the 24-hour batch. The end of the 12-hour batch, marked with the dashed line, came just as the standard deviations were starting to level out more completely. The leveling out of cross-track standard deviation at 12 hours may be due to the fact that 12 hours is the required time for each orbital plane to see every ground station. Likewise, Figure 13 is a zoomed plot showing just some of the atmospheric parameter standard deviations.

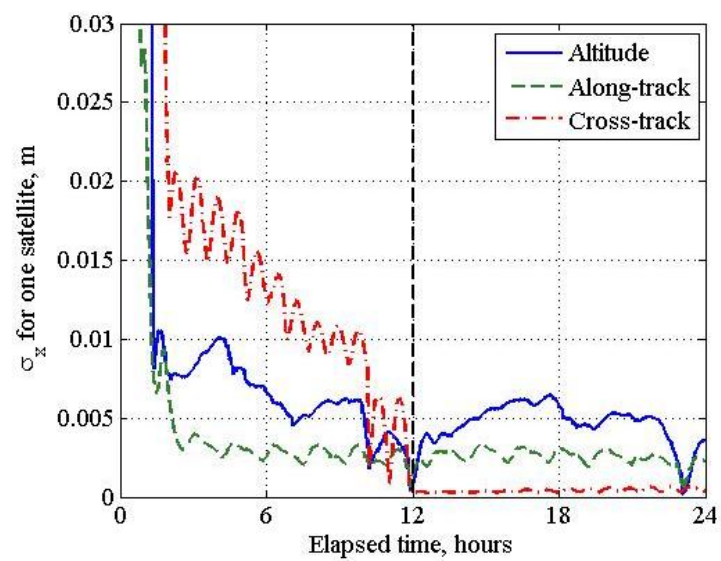

Figure 12. Position estimation error standard deviations for one satellite over 24-hour batch

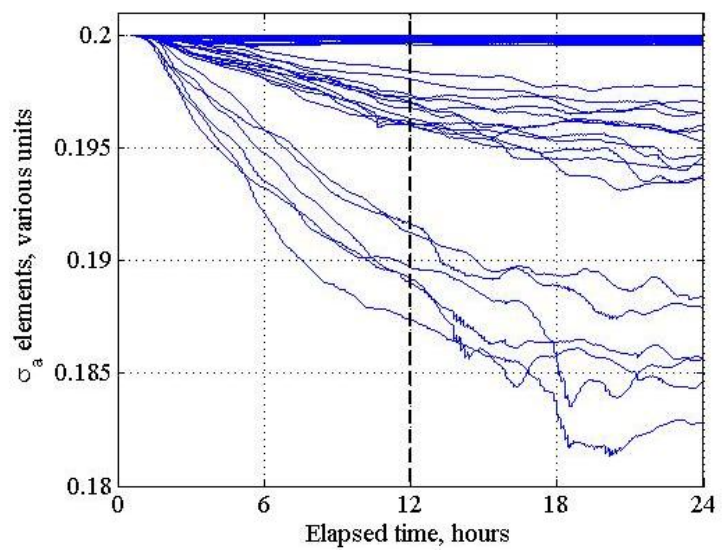

Figure 13. Atmospheric parameter estimation error standard deviations over 24-hour batch 
At the 12-hour mark, many of the uncertainties are still decreasing, but by about 20 hours they seem to reach equilibrium. Despite the extra time for the estimates to approach a steady state, the density estimate itself does not improve measurably with the doubled batch length.

For the alternate case of a lower constellation altitude described in Subsection A above, the system was expected to be more observable. This is because the combination of greater density and greater satellite velocities results in a more significant influence on satellite orbits. As Figure 14 illustrates, this was indeed the case. The standard deviations of the parameter state estimates decreased more quickly and reached lower values relative to the higheraltitude case. Oscillations at both the orbital frequency and the satellite flyby frequency are clearly visible in this data set. Figure 15 shows the mean normalized density standard deviations $\sigma_{\rho} / \rho$ at 3-hour intervals over the 12hour batch. After only 3 hours, they reached a steady state of about 0.25 , as opposed to only 0.44 for the case of higher constellation altitude.

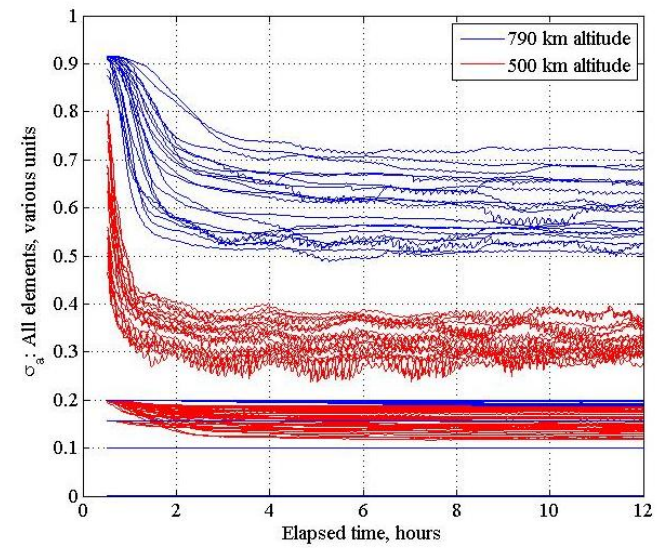

Figure 14. Comparison of estimation error standard deviations for the elements of $p_{a}$ at 790 km and $500 \mathrm{~km}$ altitudes

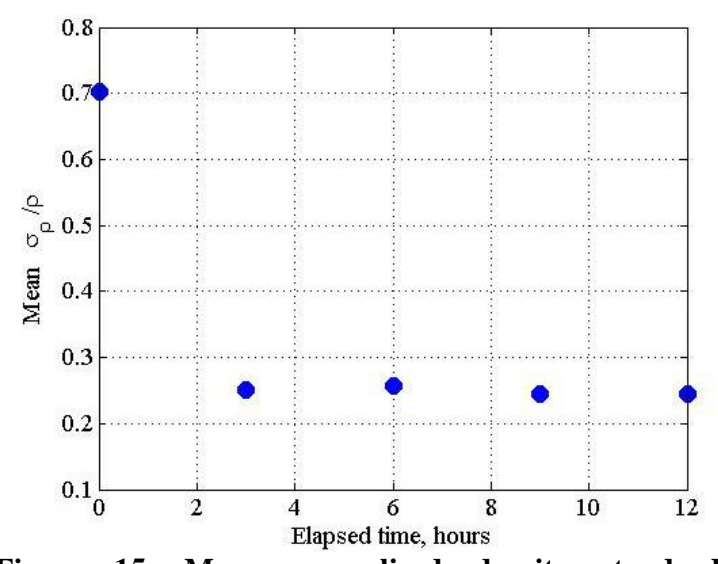

Figure 15. Mean normalized density standard deviations during 12-hour batch for $500 \mathrm{~km}$-altitude case

Although the density accuracy improved at $500 \mathrm{~km}$ altitude, Figure 16 shows that the satellite position accuracies got slightly worse. Most likely this is because the greater influence of the higher density made the orbit accuracy more susceptible to model errors and atmospheric density uncertainty. Also, each satellite at this lower altitude is tracked by the 12 ground stations less frequently, and the duration of a given pass is shorter, leading to fewer total measurements.

\section{Computational load}

Although these observability calculations have been run offline, they give an indication of whether a corresponding recursive filter could run in real-time. This is an important question given the complexity of the system. The number of satellites in the constellation and the chosen resolution of the

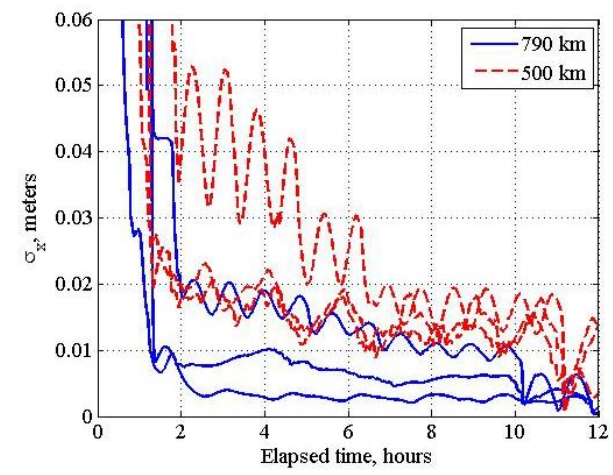

Figure 16. Comparison of one satellite's position accuracies at $790 \mathrm{~km}$ and $500 \mathrm{~km}$ altitudes atmospheric density spline dictate that the state vector contains approximately 1170 elements. At any given time the number of state vector elements depends on the number of measurement biases. Numerical integration of the satellite states and associated linearized model matrices is costly. Also of concern are the QR factorization operations that implement the covariance propagation and update steps and the necessary inversions of very large matrices. The dynamic propagation operations scale linearly as states are added, but QR factorization scales as the cube of the number of states. Calculations were performed in MATLAB on a computer with a $2.5 \mathrm{GHz}$ Intel Q9300 quad core processor and $4 \mathrm{~GB}$ of RAM, operating with 64-bit Windows Vista Business. For a typical observability analysis procedure, the dynamic propagation calculations ran for about 1.1 seconds per 5-second sample. The SRIF covariance computations took about 3.6 seconds per 5-second sample. 
In combination and with some additional overhead, the analysis ran approximately in real time. Note that the dynamic propagation calculations can be run in parallel for different satellites.

\section{E. Possible future studies}

This paper's results suggest several related avenues for future research. One would be to use real data from a satellite constellation in a filter form of this paper's algorithm. Another useful effort would re-cast the treatment of implicitly defined transmission and reception times into the framework of Ref. 29. Its framework deals with interpolation and process noise in a more rigorous manner.

\section{Summary and Conclusions}

A new atmospheric density calibration scheme has been developed that focuses on estimating a density distribution based on data from a satellite constellation. This strategy simultaneously improves the constellation orbit estimates. It leverages a rich data set of radiocommunication signals and does not require any dedicated sensors for satellite motion tracking. As part of the formulation of the estimation problem, this paper also introduces a new spline-based atmospheric density parameterization. Compared with other density representation methods, this parameterization fits more naturally into the estimation context without reducing the resolution of the density distribution.

A linearized observability analysis has been performed for the system that consists of the constellation, the radio signals, and the density distribution. This analysis aims to discover not only whether the system is theoretically observable, but also what types of assumptions or a priori knowledge might be expected to improve observability. The results show that some a priori knowledge about density is necessary to reduce uncertainty in atmospheric density estimates. Furthermore, knowledge about the satellite inverse ballistic coefficients is of limited use, because the difference in time constants tends to decouple the inverse ballistic coefficients and the density parameters if the batch is long enough. Given a reasonable initial guess of the atmospheric density spline parameters, however, the measurements do contribute to greater density estimation accuracy. Another interesting result is that the rich data set provided by the crosslink and downlink measurements makes satellite orbit accuracies relatively insensitive to some dynamic model errors. A test case with a lower constellation altitude demonstrates the effects of higher density: Due to its greater influence on the satellite orbits, density becomes more observable while decreasing the orbital position accuracies. The overall results suggest that further study of the proposed scheme for simultaneous atmospheric density calibration and constellation orbit determination would be worthwhile.

\section{Appendix}

Section II of this paper gives a conceptual overview of the spline-based atmospheric density parameterization, but it does not provide all of the details helpful for implementation. Those details are contained in this appendix. In particular, emphasis is placed on the derivation of necessary equations and on some practical implementation challenges.

\section{A. Spline equations}

As explained in Section II, the basic form of the splined parameterization is given by

$$
\rho\left(\phi, \lambda, h ; \boldsymbol{p}_{a}\right)=\rho_{0}\left(\phi, \lambda ; \boldsymbol{p}_{a}\right) \exp \left(-\frac{h-h_{0}(\phi)}{H_{\text {scale }}\left(\phi, \lambda ; \boldsymbol{p}_{a}\right)}\right)
$$

where the quantities $\rho_{0}$ and $H_{\text {scale }}$ are parameterized by two-dimension cubic spline functions. In what follows, equations are given in terms of the $\rho_{0}$ spline, but are equally applicable to any spline-parameterized quantity. The spline functions essentially work by interpolation. The parameter vector $\boldsymbol{p}_{a}$ stores $\rho_{0}$ and partial and cross-partial derivatives of $\rho_{0}$ that apply at a rectangular grid of latitude and longitude points. Values of $\rho_{0}$ at any non-grid point are obtained by interpolating between the stored values. 


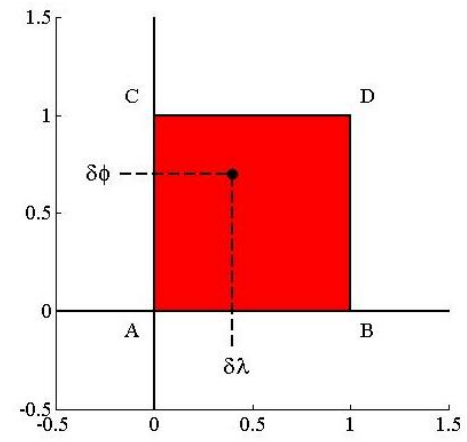

Figure A1. Basic spline function grid notation

$$
\rho_{0}(\delta \lambda, \delta \phi)=\sum_{i=0}^{3} \sum_{j=0}^{3} \alpha_{i j} \delta \lambda^{i} \delta \phi^{j}
$$

The weights $\alpha_{i j}$ are functions of the values of $\rho_{0}$ and its derivatives at the four corners of the grid cell. Suppose that the corner values are given by the vector

$$
\boldsymbol{g}=\left[\begin{array}{c}
\left(\rho_{0}\right)_{A} \\
\left(\rho_{0}\right)_{B} \\
\left(\rho_{0}\right)_{C} \\
\left(\rho_{0}\right)_{D} \\
\left(\partial \rho_{0} / \partial \delta \lambda\right)_{A} \\
\left(\partial \rho_{0} / \partial \delta \lambda\right)_{B} \\
\left(\partial \rho_{0} / \partial \delta \lambda\right)_{C} \\
\left(\partial \rho_{0} / \partial \delta \lambda\right)_{D} \\
\left(\partial \rho_{0} / \partial \delta \phi\right)_{A} \\
\left(\partial \rho_{0} / \partial \delta \phi\right)_{B} \\
\left(\partial \rho_{0} / \partial \delta \phi\right)_{C} \\
\left(\partial \rho_{0} / \partial \delta \phi\right)_{D} \\
\left(\frac{\partial^{2} \rho_{0}}{\partial \delta \lambda \partial \delta \phi}\right)_{A} \\
\left(\frac{\partial^{2} \rho_{0}}{\partial \delta \lambda \partial \delta \phi}\right)_{B} \\
\left(\frac{\partial^{2} \rho_{0}}{\partial \delta \lambda \partial \delta \phi}\right)_{C} \\
\left(\frac{\partial^{2} \rho_{0}}{\partial \delta \lambda \partial \delta \phi}\right)_{D}
\end{array}\right]
$$

Then the 16 weights $\alpha_{i j}$ can be found as a vector $\widetilde{\boldsymbol{\alpha}}$ using the equation

$$
\widetilde{\boldsymbol{\alpha}}=A^{-1} \boldsymbol{g}
$$


where the matrix $A^{-1}$ is

$$
A^{-1}=\left[\begin{array}{cccccccccccccccc}
1 & 0 & 0 & 0 & 0 & 0 & 0 & 0 & 0 & 0 & 0 & 0 & 0 & 0 & 0 & 0 \\
0 & 0 & 0 & 0 & 1 & 0 & 0 & 0 & 0 & 0 & 0 & 0 & 0 & 0 & 0 & 0 \\
-3 & 3 & 0 & 0 & -2 & -1 & 0 & 0 & 0 & 0 & 0 & 0 & 0 & 0 & 0 & 0 \\
2 & -2 & 0 & 0 & 1 & 1 & 0 & 0 & 0 & 0 & 0 & 0 & 0 & 0 & 0 & 0 \\
0 & 0 & 0 & 0 & 0 & 0 & 0 & 0 & 1 & 0 & 0 & 0 & 0 & 0 & 0 & 0 \\
0 & 0 & 0 & 0 & 0 & 0 & 0 & 0 & 0 & 0 & 0 & 0 & 1 & 0 & 0 & 0 \\
0 & 0 & 0 & 0 & 0 & 0 & 0 & 0 & -3 & 3 & 0 & 0 & -2 & -1 & 0 & 0 \\
0 & 0 & 0 & 0 & 0 & 0 & 0 & 0 & 2 & -2 & 0 & 0 & 1 & 1 & 0 & 0 \\
-3 & 0 & 3 & 0 & 0 & 0 & 0 & 0 & -2 & 0 & -1 & 0 & 0 & 0 & 0 & 0 \\
0 & 0 & 0 & 0 & -3 & 0 & 3 & 0 & 0 & 0 & 0 & 0 & -2 & 0 & -1 & 0 \\
9 & -9 & -9 & 9 & 6 & 3 & -6 & -3 & 6 & -6 & 3 & -3 & 4 & 2 & 2 & 1 \\
-6 & 6 & 6 & -6 & -3 & -3 & 3 & 3 & -4 & 4 & -2 & 2 & -2 & -2 & -1 & -1 \\
2 & 0 & -2 & 0 & 0 & 0 & 0 & 0 & 1 & 0 & 1 & 0 & 0 & 0 & 0 & 0 \\
0 & 0 & 0 & 0 & 2 & 0 & -2 & 0 & 0 & 0 & 0 & 0 & 1 & 0 & 1 & 0 \\
-6 & 6 & 6 & -6 & -4 & -2 & 4 & 2 & -3 & 3 & -3 & 3 & -2 & -1 & -2 & -1 \\
4 & -4 & -4 & 4 & 2 & 2 & -2 & -2 & 2 & -2 & 2 & -2 & 1 & 1 & 1 & 1
\end{array}\right]
$$

and the vector $\widetilde{\boldsymbol{\alpha}}$ contains the stacked scalar weights $\alpha_{i j}$ :

$$
\widetilde{\boldsymbol{\alpha}}^{\mathrm{T}}=\left[\begin{array}{llllllllllllllll}
\alpha_{00} & \alpha_{10} & \alpha_{20} & \alpha_{30} & \alpha_{01} & \alpha_{11} & \alpha_{21} & \alpha_{31} & \alpha_{02} & \alpha_{12} & \alpha_{22} & \alpha_{32} & \alpha_{03} & \alpha_{13} & \alpha_{23} & \alpha_{33}
\end{array}\right]
$$

If desired, spatial derivatives of $\rho_{0}$ are easily obtained from Eq. (A2):

$$
\begin{aligned}
\frac{\partial}{\partial \delta \lambda} \rho_{0}(\delta \lambda, \delta \phi) & =\sum_{i=0}^{3} \sum_{j=0}^{3} \alpha_{i j} i \delta \lambda^{i-1} \delta \phi^{j} \\
\frac{\partial}{\partial \delta \phi} \rho_{0}(\delta \lambda, \delta \phi) & =\sum_{i=0}^{3} \sum_{j=0}^{3} \alpha_{i j} \delta \lambda^{i} j \delta \phi^{j-1} \\
\frac{\partial^{2}}{\partial \delta \lambda \partial \delta \phi} \rho_{0}(\delta \lambda, \delta \phi) & =\sum_{i=0}^{3} \sum_{j=0}^{3} \alpha_{i j} i \delta \lambda^{i-1} j \delta \phi^{j-1}
\end{aligned}
$$

Equations (A2) and (A7) can also be written in a matrix form, which may be convenient for certain computations. To do this, create a vector of the coordinates raised to powers from 0 to 3 :

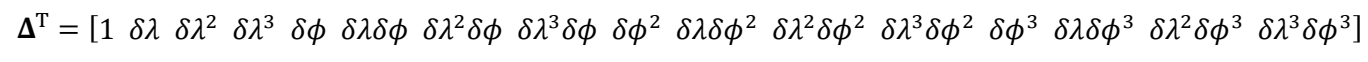

Then Eq. (A2), along with the weight vector $\widetilde{\boldsymbol{\alpha}}$ of Eq. (A4), becomes

$$
\rho_{0}(\delta \lambda, \delta \phi)=\boldsymbol{\Delta}^{\mathrm{T}} \widetilde{\boldsymbol{\alpha}}=\boldsymbol{\Delta}^{\mathrm{T}} A^{-1} \boldsymbol{g}
$$

One forms the equivalent expressions for the partial derivatives by taking derivatives of the vector $\Delta^{T}$ with respect to $\delta \lambda$ and $\delta \phi$. Derivatives with respect to true latitude and longitude (rather than normalized latitude and longitude) require a conversion from normalized grid cell units to radians.

\section{B. Constraints}

Although the spline interpolation described above works well to describe density over a rectangular region, problems arise when one applies it to a sphere or ellipsoid. As described in Section II, certain constraints are required to maintain consistency and ensure a smooth variation of density over the entire globe.

First, one must consider that longitudes of $\lambda=-90^{\circ}$ and $\lambda=90^{\circ}$ correspond to the same physical location. Consequently, $\boldsymbol{p}_{a}$ should store only one set of values for $\rho_{0}$ and its derivatives along this meridian, rather than two sets. Any computer implementation must index into the $\boldsymbol{p}_{a}$ vector in such a way that it retrieves the same values for either nominal longitude.

Second, a more complicated set of constraints arises at the two poles. Only one value of $\rho_{0}$ is stored in $\boldsymbol{p}_{a}$ for each of the poles, so that near the North pole, for instance, points $C$ and Dof Fig. A1 have the same value. This 
means that a single element of $\boldsymbol{p}_{a}$ is mapped to two elements of the vector $\boldsymbol{g}$, which is in turn used to define the weights for the cubic function.

Constraining the derivatives to ensure smooth variation of density over the polar regions requires careful thought. While all longitudes may become equivalent at these singularities, the derivatives with respect to latitude will depend on the longitude from which the pole is approached. The nature of the necessary constraints is not obvious, but the following analysis provides some clarity.

First, assume that $\rho_{0}$ is varies smoothly over the ellipsoidal surface parameterized by $\lambda$ and $\phi$. It can be equivalently written as a function of Cartesian coordinates $\rho_{0}=\rho_{0}(x, y, z)$, where $x, y$, and $z$ are related to $\lambda$ and $\phi$ by

$$
\left[\begin{array}{l}
x \\
y \\
z
\end{array}\right]=\left[\begin{array}{c}
d \cos \lambda \cos \phi \\
d \sin \lambda \cos \phi \\
\varepsilon d \sin \phi
\end{array}\right]
$$

The scalars $d$ and $\varepsilon$ determine the size and shape of the ellipsoid. Cartesian coordinates have the advantage of having no singularities at the poles.

As $\rho_{0}$ is fully differentiable, one can take derivatives with respect to $x, y$, and $z$ and then use the chain rule to compute partial derivatives with respect to $\lambda$ and $\phi$. Then one evaluates those derivatives at $\phi= \pm 90^{\circ}$. The longitude derivatives are

$$
\frac{\partial \rho_{0}}{\partial \lambda}=\frac{\partial \rho_{0}}{\partial x} \frac{\partial x}{\partial \lambda}+\frac{\partial \rho_{0}}{\partial y} \frac{\partial y}{\partial \lambda}+\frac{\partial \rho_{0}}{\partial z} \frac{\partial z}{\partial \lambda}
$$

After taking partial derivatives of the Cartesian variables according to (A10) and substituting those expressions into (A11), one obtains

$$
\frac{\partial \rho_{0}}{\partial \lambda}=-\frac{\partial \rho_{0}}{\partial x} d \sin \lambda \cos \phi+\frac{\partial \rho_{0}}{\partial y} d \cos \lambda \cos \phi+\frac{\partial \rho_{0}}{\partial z}(0)
$$

At either pole, $\cos \phi=0$, so

$$
\left.\frac{\partial \rho_{0}}{\partial \lambda}\right|_{N . P .}=\left.\frac{\partial \rho_{0}}{\partial \lambda}\right|_{S . P .}=0
$$

Next, follow the same procedure by taking latitude derivatives. First,

$$
\frac{\partial \rho_{0}}{\partial \phi}=\frac{\partial \rho_{0}}{\partial x} \frac{\partial x}{\partial \phi}+\frac{\partial \rho_{0}}{\partial y} \frac{\partial y}{\partial \phi}+\frac{\partial \rho_{0}}{\partial z} \frac{\partial z}{\partial \phi}
$$

Substituting the derivatives of Eq. (A10), (A14) becomes

$$
\frac{\partial \rho_{0}}{\partial \phi}=-\frac{\partial \rho_{0}}{\partial x} d \cos \lambda \sin \phi-\frac{\partial \rho_{0}}{\partial y} d \sin \lambda \sin \phi+\frac{\partial \rho_{0}}{\partial z} \varepsilon d \cos \phi
$$

When (A15) is evaluated at each pole, the result is

$$
\begin{aligned}
& \left.\frac{\partial \rho_{0}}{\partial \phi}\right|_{N . P .}=-d\left[\frac{\partial \rho_{0}}{\partial x} \cos \lambda+\frac{\partial \rho_{0}}{\partial y} \sin \lambda\right] \\
& \left.\frac{\partial \rho_{0}}{\partial \phi}\right|_{S . P .}=+d\left[\frac{\partial \rho_{0}}{\partial x} \cos \lambda+\frac{\partial \rho_{0}}{\partial y} \sin \lambda\right]
\end{aligned}
$$


Finally, the same process is applied to calculate the cross-partial derivatives with respect to latitude and longitude. After some algebra, and recalling that $\cos \phi=0$, all the higher order derivatives with respect to the Cartesian variables vanish. The remaining non-zero expressions are

$$
\begin{aligned}
& \left.\frac{\partial^{2} \rho_{0}}{\partial \lambda \partial \phi}\right|_{N . P .}=+d\left[\frac{\partial \rho_{0}}{\partial x} \sin \lambda-\frac{\partial \rho_{0}}{\partial y} \cos \lambda\right] \\
& \left.\frac{\partial^{2} \rho_{0}}{\partial \lambda \partial \phi}\right|_{\text {S.P. }}=-d\left[\frac{\partial \rho_{0}}{\partial x} \sin \lambda-\frac{\partial \rho_{0}}{\partial y} \cos \lambda\right]
\end{aligned}
$$

It should be noted that Eqs. (A17) are very similar in form to Eqs. (A16). This similarity can be stated more explicitly by defining some new variables:

$$
\begin{aligned}
& P_{A_{N}}=\left.d \frac{\partial \rho_{0}}{\partial x}\right|_{N . P .} . \\
& P_{B_{N}}=\left.d \frac{\partial \rho_{0}}{\partial y}\right|_{N . P .} \\
& P_{A_{S}}=\left.d \frac{\partial \rho_{0}}{\partial x}\right|_{S . P .} \\
& P_{B_{S}}=\left.d \frac{\partial \rho_{0}}{\partial y}\right|_{\text {S.P. }}
\end{aligned}
$$

After substituting Eqs. (A18) into Eqs. (A16) and (A17), one obtains

$$
\begin{aligned}
& \left.\frac{\partial \rho_{0}}{\partial \phi}\right|_{N . P .}=-P_{A_{N}} \cos \lambda-P_{B_{N}} \sin \lambda \\
& \left.\frac{\partial \rho_{0}}{\partial \phi}\right|_{S . P .}=P_{A_{S}} \cos \lambda+P_{B_{S}} \sin \lambda \\
& \left.\frac{\partial^{2} \rho_{0}}{\partial \lambda \partial \phi}\right|_{N . P .}=P_{A_{N}} \sin \lambda-P_{B_{N}} \cos \lambda \\
& \left.\frac{\partial^{2} \rho_{0}}{\partial \lambda \partial \phi}\right|_{S . P .}=-P_{A_{S}} \sin \lambda+P_{B_{S}} \cos \lambda
\end{aligned}
$$

The key result, as expressed in Eqs. (A19), is that all the partial derivatives at the poles are completely specified by just two parameters per pole. For a given longitude, the standard spline-form derivatives can be determined from these two parameters, which are thus the only quantities stored in the vector $\boldsymbol{p}_{a}$ to represent all the partial derivatives at each pole. This result makes sense if one considers what it means for $\rho_{0}$ to be differentiable at the poles: in a sufficiently small region surrounding each pole, one can think of $\rho_{0}$ as locally looking like a flat plane, which is completely described by its tilt direction and angle.

\section{Parameter transformation}

Although to this point the splined atmosphere representation has been described in terms of the quantities $\rho_{0}$ and $H_{\text {scale }}$, it is really only correct to say that the two sets of cubic spline values encode the information about these quantities. In actual implementation, the vector $\boldsymbol{p}_{a}$ contains values of $\log \left(\rho_{0}\right)$ and $1 / H_{\text {scale }}$ and the derivatives of those quantities rather than $\rho_{0}$ and $H_{\text {scale }}$ directly.

There are several reasons for these nonlinear transformations. First, physical density can never be negative, but standard estimation procedures provide no easy way to prevent estimates from becoming negative if inaccurate measurements so indicate. By calculating density as $\rho=e^{\log (\rho)}$, this situation is averted. Second, the logarithmic transformation makes the two spline functions add together linearly, which aids computational ease significantly. To demonstrate this, take the natural logarithm of both sides of Eq. (A1):

$$
\log (\rho)=\log \left(\rho_{0} \exp \left(-\frac{h-h_{0}}{H_{\text {scale }}}\right)\right)=\log \left(\rho_{0}\right)-\left(h-h_{0}\right) \frac{1}{H_{\text {scale }}}
$$


While it is relatively easy to move between $\rho_{0}$ and $\log \left(\rho_{0}\right)$, or between $H_{\text {scale }}$ and $\frac{1}{H_{\text {scale }}}$, the transformation of the derivatives is slightly more complicated. By applying the chain rule, one can derive the relationships

$$
\begin{gathered}
\frac{\partial \log \left(\rho_{0}\right)}{\partial \phi}=\frac{\partial \log \left(\rho_{0}\right)}{\partial \rho_{0}} \frac{\partial \rho_{0}}{\partial \phi}=\frac{1}{\rho_{0}} \frac{\partial \rho_{0}}{\partial \phi} \\
\frac{\partial \log \left(\rho_{0}\right)}{\partial \lambda}=\frac{\partial \log \left(\rho_{0}\right)}{\partial \rho_{0}} \frac{\partial \rho_{0}}{\partial \lambda}=\frac{1}{\rho_{0}} \frac{\partial \rho_{0}}{\partial \lambda} \\
\frac{\partial^{2} \log \left(\rho_{0}\right)}{\partial \lambda \partial \phi}=\frac{\partial}{\partial \lambda}\left(\frac{1}{\rho_{0}} \frac{\partial \rho_{0}}{\partial \phi}\right)=\frac{\partial}{\partial \rho_{0}}\left(\frac{1}{\rho_{0}}\right) \frac{\partial \rho_{0}}{\partial \lambda} \frac{\partial \rho_{0}}{\partial \phi}+\frac{1}{\rho_{0}} \frac{\partial^{2} \rho_{0}}{\partial \lambda \partial \phi}=-\frac{1}{\rho_{0} 2} \frac{\partial \rho_{0}}{\partial \lambda} \frac{\partial \rho_{0}}{\partial \phi}+\frac{1}{\rho_{0}} \frac{\partial^{2} \rho_{0}}{\partial \lambda \partial \phi}
\end{gathered}
$$

for the derivatives of $\log \left(\rho_{0}\right)$, and

$$
\begin{aligned}
\frac{\partial}{\partial \phi}\left(\frac{1}{H_{\text {scale }}}\right) & =-\frac{1}{H_{\text {scale }}^{2}} \frac{\partial H_{\text {scale }}}{\partial \phi} \\
\frac{\partial}{\partial \lambda}\left(\frac{1}{H_{\text {scale }}}\right) & =-\frac{1}{H_{\text {scale }}^{2}} \frac{\partial H_{\text {scale }}}{\partial \lambda} \\
\frac{\partial^{2}}{\partial \lambda \partial \phi}\left(\frac{1}{H_{\text {scale }}}\right)=\frac{\partial}{\partial \lambda}\left(-\frac{1}{H_{\text {scale }}^{2}} \frac{\partial H_{\text {scale }}}{\partial \phi}\right) & =\frac{2}{H_{\text {scale }}{ }^{3}} \frac{\partial H_{\text {scale }}}{\partial \lambda} \frac{\partial H_{\text {scale }}}{\partial \phi}-\frac{1}{H_{\text {scale }}{ }^{2}} \frac{\partial^{2} H_{\text {scale }}}{\partial \lambda \partial \phi}
\end{aligned}
$$

for the derivatives of $1 / H_{\text {scale }}$.

One major challenge associated with this nonlinear transformation is the specification of appropriate time constants and Markov process standard deviations for the elements of the parameter vector $\boldsymbol{p}_{a}$. For instance, it is relatively easy to determine a reasonable value for the standard deviation of $\rho$ at a particular place or time. It is much more difficult to determine the corresponding appropriate standard deviation for $\partial \log \left(\rho_{0}\right) / \partial \phi$ that will yield the desired standard deviation for $\rho$.

\section{Initialization procedure}

The initialization procedure described in Section II is really just the inverse of the problem of density calculation. The forward problem seeks the value of density at a given location as a function of the spline values at the grid cell corners; the inverse problem seeks the values of the spline function at the grid points that would most nearly cause the density to match a set of "measurements". If there are enough independent measurements of density, then many instances of Eq. (A9) can be stacked like a batch of measurements:

$$
\left[\begin{array}{c}
\rho_{1} \\
\rho_{2} \\
\vdots \\
\rho_{N}
\end{array}\right]=\left[\begin{array}{c}
\Delta_{1}^{\mathrm{T}} \\
\boldsymbol{\Delta}_{2}^{\mathrm{T}} \\
\vdots \\
\boldsymbol{\Delta}_{N}^{\mathrm{T}}
\end{array}\right] A^{-1} \boldsymbol{g}
$$

This expression can then be solved for $\boldsymbol{g}$ in a least-squares sense to obtain the grid point values that would yield the measured densities.

$$
\boldsymbol{g}=A\left(\left[\begin{array}{c}
\boldsymbol{\Delta}_{1}^{\mathrm{T}} \\
\boldsymbol{\Delta}_{2}^{\mathrm{T}} \\
\vdots \\
\boldsymbol{\Delta}_{N}^{\mathrm{T}}
\end{array}\right]^{\mathrm{T}}\left[\begin{array}{c}
\boldsymbol{\Delta}_{1}^{\mathrm{T}} \\
\boldsymbol{\Delta}_{2}^{\mathrm{T}} \\
\vdots \\
\boldsymbol{\Delta}_{N}^{\mathrm{T}}
\end{array}\right]\right)^{-1}\left[\begin{array}{c}
\boldsymbol{\Delta}_{1}^{\mathrm{T}} \\
\boldsymbol{\Delta}_{2}^{\mathrm{T}} \\
\vdots \\
\boldsymbol{\Delta}_{N}^{\mathrm{T}}
\end{array}\right]\left[\begin{array}{c}
\rho_{1} \\
\rho_{2} \\
\vdots \\
\rho_{N}
\end{array}\right]
$$

The initialization procedure obtains pseudo-measurements of density from an existing atmospheric model and solves for the vector $\boldsymbol{p}_{a}$, which contains the spline corner values for not just one grid cell at a time but all of them at once. The basic least-squares problem is the same as that of Eq. (24), but some careful indexing is required to refer each pseudo-measurement to the appropriate sub-vector of $\boldsymbol{p}_{a}$. Also, the actual initialization procedure works with the spline functions for $\log \left(\rho_{0}\right)$ and $1 / H_{\text {scale }}$, but still follows the conceptual algorithm presented here. 


\section{E. Partial derivatives}

As discussed in Section II, the estimation problem requires the partial derivatives $\frac{\partial \rho}{\partial r_{I C R S}}$ and $\frac{\partial \rho}{\partial \boldsymbol{p}_{a}}$. Equation (A7) readily provides $\frac{\partial \rho_{0}}{\partial \phi}, \frac{\partial H_{\text {scale }}}{\partial \phi}, \frac{\partial \rho_{0}}{\partial \lambda}$, and $\frac{\partial H_{\text {scale }}}{\partial \lambda}$. Differentiation of a modified form of Eq. (A23) gives $\frac{\partial \rho_{0}}{\partial p_{a}}$ and $\frac{\partial H_{s c a l e}}{\partial \boldsymbol{p}_{a}}$. The required derivatives can be computed from the available ones by repeated application of the chain rule. For $\frac{\partial \rho}{\partial \boldsymbol{p}_{a}}$, this is a relatively simple procedure involving some minor algebraic manipulation. The final result is

$$
\frac{\partial \rho}{\partial \boldsymbol{p}_{a}}=\frac{\partial \rho_{0}}{\partial \boldsymbol{p}_{a}} \frac{\rho}{\rho_{0}}-\frac{\rho}{H_{\text {scale }}}\left(\log \left(\frac{\rho}{\rho_{0}}\right) \frac{\partial H_{\text {scale }}}{\partial \boldsymbol{p}_{a}}\right)
$$

In order to compute $\frac{\partial \rho}{\partial r_{I C R S}}$, the partial derivative of density with respect to inertial position, one first finds derivatives of density with respect to latitude, longitude, and altitude. Starting with Eq. (A1) and after some straightforward differentiation and algebra, these expressions are

$$
\begin{gathered}
\frac{\partial \rho}{\partial \phi}=\frac{\partial \rho_{0}}{\partial \phi} \frac{\rho}{\rho_{0}}+\frac{\rho}{H_{\text {scale }}}\left(\frac{\partial h_{0}}{\partial \phi}-\log \left(\frac{\rho}{\rho_{0}}\right) \frac{\partial H_{\text {scale }}}{\partial \phi}\right) \\
\frac{\partial \rho}{\partial \lambda}=\frac{\partial \rho_{0}}{\partial \lambda} \frac{\rho}{\rho_{0}}-\frac{\rho}{H_{\text {scale }}}\left(\log \left(\frac{\rho}{\rho_{0}}\right) \frac{\partial H_{\text {scale }}}{\partial \lambda}\right) \\
\frac{\partial \rho}{\partial h}=-\frac{\rho}{H_{\text {scale }}}
\end{gathered}
$$

Note that the constellation nominal altitude $h_{0}$ is a function of latitude because it depends on the local radius of the WGS-84 ellipsoid. Its derivative $\frac{\partial h_{0}}{\partial \phi}$ can be obtained by differentiating the ellipsoid equations.

The next step involves the derivatives that transform from latitude, longitude, and altitude to an Earth-fixed position, and then from Earth-fixed coordinates to inertial coordinates. The resulting expression is

$$
\frac{\partial \rho}{\partial r_{I C R S}^{s a t}}=\left(\frac{\partial \rho}{\partial \phi} \frac{\partial \phi}{\partial r_{I T R S}^{s a t}}+\frac{\partial \rho}{\partial \lambda} \frac{\partial \lambda}{\partial r_{I T R S}^{s a t}}+\frac{\partial \rho}{\partial h} \frac{\partial h}{\partial r_{I T R S}^{s a t}}\right) A_{r o t}^{\mathrm{T}}(t)
$$

where $\frac{\partial \phi}{\partial r_{I T R S}^{\text {sat }}}, \frac{\partial \lambda}{\partial r_{I T R S}^{\text {sat }}}$, and $\frac{\partial h}{\partial r_{I T R S}^{\text {sat }}}$ are assumed to be available from the algorithm that converts latitude, longitude, and altitude to a Cartesian position vector, and $A_{\text {rot }}(t)$ is the time-varying rotation matrix that transforms between the Earth-fixed and inertial coordinate systems.

\section{References}

${ }^{1}$ Puderbaugh, A.L., Dixon, G.L., Jr., Shroyer, L.E., Boyce, W.H. III, and Shepperd, R.W., “A Global and Local History of Drag Effects at Iridium Mission Altitude," Proc. of AIAA/AAS Astrodynamics Specialist Conference and Exhibit, 5-8 August 2002, Monterey, CA, AIAA 2002-4733 (2002).

2 Jacchia, L.G., "Density Variations in the Heterosphere," Smithsonian Astrophysical Observatory Special Report \#184, Cambridge, MA, 1965.

${ }^{3}$ Jacchia, L.G., "New Static Models of the Thermosphere and Exosphere with Empirical Temperature Profiles," Smithsonian Astrophysical Observatory Special Report \#313, Cambridge, MA, 1970.

${ }^{4}$ Jacchia, L.G., "Thermospheric Temperature, Density, and Composition: New Models," Smithsonian Astrophysical Observatory Special Report \#375, Cambridge, MA, 1977.

${ }^{5}$ Roberts, C.E., “An Analytical Model for Upper Atmosphere Densities Based Upon Jacchia's 1970 Models,” Celestial Mechanics, Vol. 4, December 1971, pp. 368-377.

${ }^{6}$ Hedin, A.E., "The Atmospheric Model in the Region 90 to 2000 km," Adv. in Space Research, Vol. 8, No. 5, 1988, pp. 925.

${ }^{7}$ Hedin, A.E., "Extension of the MSIS Thermosphere Model into the Middle and Lower Atmosphere," J. of Geophysical Research, Vol. 96, No. A2, February 1991, pp. 1159-1172.

${ }^{8}$ Picone, J.M., Hedin, A.E., Drob, D.P., and Aikin, A.C., "NRLMSISE-00 Empirical Model of the Atmosphere: Statistical Comparisons and Scientific Issues,” J. of Geophysical Research, Vol. 107, No. A12, 2002, pp. 1468-1483.

${ }^{9}$ Marcos, F.A., Wise, J.O., Kendra, M.J., and Grossbard, N., "Advances in Satellite Drag Modeling," Proc. of 42 ${ }^{\text {nd }}$ AIAA Aerospace Sciences Meeting and Exhibit, 5-8 January 2004, Reno, NV, AIAA 2004-1254 (2004). 
${ }^{10}$ Lean, J., Picone, J.M., Knowles, S., Hedin, A., and Moore, G., "Validating NRLMSIS Using Atmospheric Densities Derived from Spacecraft Drag: Starshine Example," Proc. of AIAA/AAS Astrodynamics Specialist Conference and Exhibit, 5-8 August 2002, Monterey, CA, AIAA 2002-4736 (2002).

${ }^{11}$ Pardini, C. and Anselmo, L., "Comparison and Accuracy Assessment of Semi-empirical Atmosphere Models through the Orbital Decay of Spherical Satellites," J. of the Astronautical Sciences, Vol. 49, No. 2, 2001, pp. 255-268.

${ }^{12}$ Marcos, F.A., Kendra, M.J., Griffin, J.M., Bass, J.N., Larson, D.R., and Liu, J.J., "Precision Low Earth Orbit Determination Using Atmospheric Density Calibration," J. of the Astronautical Sciences, Vol. 46, No. 4, Oct.-Dec. 1998, pp. 395-409.

${ }^{13}$ Marcos, F.A., Kendra, M.J., and Bass, J.N., "Recent Advances in Satellite Drag Modeling," Proc. of 37 $7^{\text {th }}$ AIAA Aerospace Sciences Meeting and Exhibit, 11-14 January 1999, Reno, NV, AIAA 99-0631 (1999).

${ }^{14}$ Bowman, B.R., Tobiska, W.K., Marcos, F.A., Huang, C.Y., Lin, C.S., and Burke, W.J., “A New Empirical Density Model JB2008 Using New Solar and Geomagnetic Indices," Proc. of AIAA/AAS Astrodynamics Specialist Conference, 18-21 August 2008, Honolulu, HI, AIAA 2008-6438 (2008).

${ }^{15}$ Hedin, A.E., and Carignan, G.R., "Morphology of Thermospheric Composition Variations in the Quiet Polar Thermosphere From Dynamics Explorer Measurements," J. of Geophysical Research, Vol. 90, No. A6, June 1985, pp 52695277.

${ }^{16}$ Yunck, T.P., "Coping with the Atmosphere and Ionosphere in Precise Satellite and Ground Positioning," Geophysical Monograph Series, Vol. 73, A. Jones (ed.), American Geophysical Union, Washington, D.C., 1993, pp. 1-16.

${ }^{17}$ DeBra, D.B., "Drag-Free Spacecraft as Platforms for Space Missions and Fundamental Physics," Class. Quantum Grav., Vol. 14, 1997, pp. 1549-1555.

${ }^{18}$ Nazarenko, A.I., Cefola, P.J., and Yurasov, V., "Estimating Atmospheric Density Variations to Improve LEO Orbit Prediction Accuracy," Proc. of the AAS/AIAA Spaceflight Mechanics Meeting, 1998, pp 1235-1264.

${ }^{19}$ Yurasov, V.S., Nazarenko, A.I., Alfriend, K.T., and Cefola, P.J., "Direct Density Correction Method: Review of Results," Proc. of 57th Intl. Astronautical Conf., Valencia, Spain, 2-6 October 2006.

${ }^{20}$ Picone, J.M., Emmert, J.T., and Lean, J.L., "Thermospheric Densities Derived from Spacecraft Orbits: Accurate Processing of Two-Line Element Sets," J. of Geophysical Research, Vol. 110, No. A03301, 2005.

${ }^{21}$ Doornbos, E., Klinkrad, H., and Visser, P., "Use of Two-Line Element Data for Thermosphere Neutral Density Model Calibration," Adv. in Space Research, Vol. 41, 2008, pp. 1115-1122.

${ }^{22}$ Casali, S.J., and Barker, W.N., "Dynamic Calibration Atmosphere (DCA) for the High Accuracy Satellite Drag Model (HASDM)," Proc. of Astrodynamics Specialist Conference and Exhibit, 5-8 August 2002, Monterey, CA, AIAA $2002-4888$ (2002).

${ }^{23}$ Storz, M.F., Bowman, B.R., Branson, Major J.I., Casali, S.J., and Tobiska, W.K., "High Accuracy Satellite Drag Model (HASDM)," Adv. in Space Research, Vol. 36, 2005, pp. 2497-2505.

${ }^{24}$ Anderson, R.L., Born, G.H., and Forbes, J.M., "Sensitivity of Orbit Predictions to Density Variability," J.of Spacecraft and Rockets, Vol. 46, No. 6, 2009, pp. 1214-1230.

${ }^{25}$ Psiaki, M.L., “Autonomous Low-Earth-Orbit Determination from Magnetometer and Sun Sensor Data," J.of Guidance, Control, and Dynamics, Vol. 22, No. 2, March-April 1999, pp. 296-304.

${ }^{26}$ Brown, R.G. and Hwang, P.Y.C., Introduction to Random Signals and Applied Kalman Filtering with Matlab Exercises and Solutions, John Wiley \& Sons, $3^{\text {rd }}$ Ed., New York (1997), pp. 428-431.

${ }^{27}$ Wright, J.R., and Woodburn, J., "Simultaneous Real-Time Estimation of Atmospheric Density and Ballistic Coefficient," Paper AAS 04-175, Spaceflight Mechanics 2003-Adv. in the Astronautical Sciences, Vol. 114, pp. 927-950, Proc. of the 13 ${ }^{\text {th }}$ AAS/AIAA Space Flight Mechanics Conf., Ponce, Puerto Rico, 9-13 February 2003.

${ }^{28}$ Wright, J.R., "Real-Time Estimation of Local Atmospheric Density," Paper AAS 03-164, Spaceflight Mechanics 2005Adv. in the Astronautical Sciences, Vol. 119, pp. 1155-1184, Proc. of the $14^{\text {th }}$ AAS/AIAA Space Flight Mechanics Conf., Maui, HI, 8-12 February 2004.

${ }^{29}$ Mohiuddin. S., and Psiaki, M.L., "Continuous-Time Kalman Filtering with Implicit Discrete Measurement Times," submitted to the Journal of Guidance, Control, \& Dynamics, in review.

${ }^{30}$ Mitch, R.H., and Psiaki, M.L., "Local Ionosphere Model Estimation from Dual-Frequency GNSS TEC Data," to be presented at ION GNSS 2010, Portland, OR, 21-24 September 2010.

${ }^{31}$ Bierman, G.J., Factorization Methods for Discrete Sequential Estimation, Academic Press, New York, 115-122 (1977), pp. 69-76.

${ }^{32}$ Psiaki, M.L., and Hinks, J.C., “Autonomous Lunar Orbit Determination using Star Occultation Measurements,” Proc. of AIAA Guidance, Navigation, and Control Conference and Exhibit, 20-23 August 2007, Hilton Head, SC, AIAA $2007-6657$ (2007). 